\title{
Nonconservative Diffusions on $[0,1]$ with Killing and Branching: Applications to Wright-Fisher Models with or without Selection
}

\author{
Thierry E. Huillet \\ Laboratoire de Physique Théorique et Modélisation, CNRS-UMR 8089 et Université de Cergy-Pontoise, \\ 2 Avenue Adolphe Chauvin, 95302 Cergy-Pontoise, France \\ Correspondence should be addressed to Thierry E. Huillet, thierry.huillet@u-cergy.fr
}

Received 22 November 2010; Accepted 9 May 2011

Academic Editor: Manuel O. Cáceres

Copyright (C) 2011 Thierry E. Huillet. This is an open access article distributed under the Creative Commons Attribution License, which permits unrestricted use, distribution, and reproduction in any medium, provided the original work is properly cited.

\begin{abstract}
We consider nonconservative diffusion processes $x_{t}$ on the unit interval, so with absorbing barriers. Using Doob-transformation techniques involving superharmonic functions, we modify the original process to form a new diffusion process $\tilde{x}_{t}$ presenting an additional killing rate part $d>0$. We limit ourselves to situations for which $\tilde{x}_{t}$ is itself nonconservative with upper bounded killing rate. For this transformed process, we study various conditionings on events pertaining to both the killing and the absorption times. We introduce the idea of a reciprocal Doob transform: we start from the process $\tilde{x}_{t}$, apply the reciprocal Doob transform ending up in a new process which is $x_{t}$ but now with an additional branching rate $b>0$, which is also upper bounded. For this supercritical binary branching diffusion, there is a tradeoff between branching events giving birth to new particles and absorption at the boundaries, killing the particles. Under our assumptions, the branching diffusion process gets eventually globally extinct in finite time. We apply these ideas to diffusion processes arising in population genetics. In this setup, the process $x_{t}$ is a Wright-Fisher diffusion with selection. Using an exponential Doob transform, we end up with a killed neutral Wright-Fisher diffusion $\tilde{x}_{t}$. We give a detailed study of the binary branching diffusion process obtained by using the corresponding reciprocal Doob transform.
\end{abstract}

\section{Introduction}

We consider diffusion processes on the unit interval with a series of elementary stochastic models arising chiefly in population dynamics in mind. These connections found their way over the last sixty years, chiefly in mathematical population genetics. In this context, we refer to [1] and to its extensive and nonexhaustive list of references for historical issues in the development of modern mathematical population genetics (after Wright, Fisher, Crow, Kimura, Nagylaki, Maruyama, Ohta, Watterson, Ewens, Kingman, Griffiths, and Tavaré, to cite only a few). See also the general monographs [2-6]. 
Special emphasis is put on Doob-transformation techniques of the diffusion processes under concern. Most of the paper's content focuses on the specific Wright-Fisher (WF) diffusion model and some of its variations, describing the evolution of one two-locus colony undergoing random mating, possibly under the additional actions of mutation, selection, and so on. We now describe the content of this work in more detail.

Section 2 is devoted to generalities on one-dimensional diffusions on the unit interval $[0,1]$. It is designed to fix the background and notations. Special emphasis is put on the Kolmogorov backward and forward equations, while stressing the crucial role played by the boundaries in such one-dimensional diffusion problems. Some questions such as the meaning of speed and scale functions, existence of an invariant measure, and validity of detailed balance are addressed in the light of the Feller classification of boundaries. When the boundaries are absorbing, the important problem of evaluating additive functionals along sample paths is then briefly discussed, emphasizing the prominent role played by the Green function of the model; several simple illustrative examples are supplied. So far, we have dealt with a given process, say $x_{t}$, and recalled the various ingredients for computing the expectations of various quantities of interest, summing up over the history of paths. In this setup, there is no distinction among paths with different destinations, nor did we allow for annihilation or creation of paths inside the domain before the process reached one of the boundaries. The Doob transform of paths allows to do so. We, therefore, describe the transformation of sample paths techniques deriving from superharmonic additive functionals. Some Doob transformations of interest are then investigated, together with the problem of evaluating additive functionals of the transformed diffusion process itself. Roughly speaking, the transformation of paths procedure allows to select sample paths of the original process with, say, a fixed destination and/or, more generally, to kill certain sample paths that do not fit the integral criterion encoded by the additive functional. As a result, this selection of paths procedure leads to a new process described by an appropriate modification of the infinitesimal generator of the original process including a multiplicative killing part rate of the sample paths inside the interval. It turns out, therefore, that the same diffusion methods used in the previous discussions apply to the transformed processes, obtained after a change of measure.

Let us be more specific. In this work, we limit ourselves to nonconservative diffusion processes $x_{t}$ on the unit interval and so with absorbing barriers. Using Doob-transformation techniques involving superharmonic functions $\alpha$, we modify the original process to form a new diffusion process $\tilde{x}_{t}$ presenting an additional killing rate part $d>0$. We further limit ourselves to situations for which $\widetilde{x}_{t}$ is itself nonconservative with bounded above killing rate. For a large class of diffusion processes, the exponential function or some linear combinations of exponential functions are admissible superharmonic functions $\alpha$, leading to the required property on $d$. The full transformed process has two stopping times: the time to absorption to the boundaries and the killing time inside the domain. We study various conditionings of the transformed process: conditioning on events leading to both random stopping times occurring after the current time or only in the remote future and conditioning on events leading to either killing or absorption time occurring first. We give the relevant quasistationary limit laws, in the spirit of Yaglom [7]. This is made possible thanks to the existence of an harmonic function for the full infinitesimal generator of the transformed process.

We next introduce the idea of a reciprocal Doob transform: we start now from the process $\tilde{x}_{t}$, apply the reciprocal Doob transform ending up in a new process which is $x_{t}$ but now with an additional branching rate $b>0$, which is bounded. Under this reciprocal technique, the particles are not killed, rather they are allowed either to survive or split. The 
transformed process is a binary branching diffusion. For this supercritical binary branching diffusion process, there is a tradeoff between branching events giving birth to new particles and absorption at the boundaries, killing the particles. Under our assumptions, the branching diffusion process gets eventually globally extinct in finite time.

We next apply these general ideas to diffusion processes arising in population genetics.

In Section 3 we start recalling that Wright-Fisher diffusion models with various drifts are continuous space-time models which can be obtained as scaling limits of a biased discrete Galton-Watson model with a conservative number of offspring over the generations. Sections 4 and 5 are devoted to a detailed study of both the neutral WF diffusion process and the WF diffusion with selection, respectively.

In Section 6, we apply the Doob-transformation techniques to these processes: The starting point process $x_{t}$ is a Wright-Fisher diffusion with selection differential $\sigma>0$. We use the exponential Doob kernel $\alpha=e^{-\sigma x}$. The transformed process accounts for a neutral Wright-Fisher evolution $\tilde{x}_{t}$ for the allele 1 frequency, subject to the additional possibility of the extinction of the population itself due to killing at rate $d$ proportional to its heterozygosity. This model is of importance in population genetics as it first appeared in [8, Page 272] as a scaling limit of a discrete population genetics model of recombination. We particularize the relevant Yaglom limit laws obtained after conditionings on events pertaining to both the killing or the absorption times occurring first. The computations of the quasistationary distributions are explicit here. Our approach relies on the spectral expansion of the transition probability kernels of both $x_{t}$ and $\tilde{x}_{t}$ which are known (from the works of Kimura) to involve oblate spheroidal wave functions and Gegenbauer polynomials, respectively.

In Section 7, we follow the general reciprocal path indicated in Section 2 and apply it to the particular models under concern, thereby illustrating and developing the idea of a reciprocal Doob transform. We give a detailed study of the binary branching diffusion process obtained by using the corresponding reciprocal Doob transform $e^{\sigma x}$ when the starting point process is now a neutral Wright-Fisher diffusion process. We end up in a globally subcritical branching particle system, each diffusing according to the WF model with selection. This problem is amenable to the results obtained in $[9,10]$.

\section{Diffusion Processes on The Unit Interval: A Reminder}

We start with generalities on one-dimensional diffusions exemplifying our study to the Wright-Fisher model and its relatives. For more technical details, we refer to [8, 11-13].

\subsection{Generalities on One-Dimensional Diffusions on the Interval $[0,1]$}

Let $\left(w_{t} ; t \geq 0\right)$ be a standard one-dimensional Brownian (Wiener) motion. Consider a onedimensional Itô diffusion driven by $\left(w_{t} ; t \geq 0\right)$ on the interval say $[0,1]$; see [14]. We will let $I=(0,1)$. Assume that it has locally Lipschitz continuous drift $(x)$ and local standard deviation (volatility) $g(x)$, namely, consider the stochastic differential equation (SDE)

$$
d x_{t}=f\left(x_{t}\right) d t+g\left(x_{t}\right) d w_{t}, \quad x_{0}=x \in(0,1)
$$

The condition on $f(x)$ and $g(x)$ guarantees in particular that there is no point $x_{*}$ in $I$ for which $|f(x)|$ or $|g(x)|$ would blow up and diverge as $\left|x-x_{*}\right| \rightarrow 0$. 
The Kolmogorov backward infinitesimal generator of (2.1) is $G=f(x) \partial_{x}+(1 / 2)$ $g^{2}(x) \partial_{x}^{2}$. As a result, for all suitable $\psi$ in the domain of the operator $S_{t}:=e^{t G}, u:=u(x, t)=$ $\mathrm{E} \psi\left(x_{t \wedge \tau_{x}}\right)$ satisfies the Kolmogorov backward equation (KBE)

$$
\partial_{t} u=G(u) ; \quad u(x, 0)=\psi(x) .
$$

In the definition of the mathematical expectation $u$, we have $t \wedge \tau_{x}:=\inf \left(t, \tau_{x}\right)$, where $\tau_{x}$ indicates a random time at which the process should possibly be stopped (absorbed), given the process was started in $x$. The description of this (adapted) absorption time is governed by the type of boundaries which $\{0,1\}$ are to $\left(x_{t} ; t \geq 0\right)$.

\subsection{Natural Coordinate, Scale, and Speed Measure}

For such Markovian diffusions, it is interesting to consider the $G$-harmonic coordinate $\varphi \in C^{2}$ belonging to the kernel of $G$ that is, satisfying $G(\varphi)=0$. For $\varphi$ and its derivative $\varphi^{\prime}:=d \varphi / d y$, with $\left(x_{0}, y_{0}\right) \in(0,1)$, one finds

$$
\begin{gathered}
\varphi^{\prime}(y)=\varphi^{\prime}\left(y_{0}\right) e^{-2 \int_{y_{0}}^{y}\left(f(z) /\left(g^{2}(z)\right)\right) d z} \\
\varphi(x)=\varphi\left(x_{0}\right)+\varphi^{\prime}\left(y_{0}\right) \int_{x_{0}}^{x} e^{-2 \int_{y_{0}}^{y}\left(f(z) /\left(g^{2}(z)\right)\right) d z} d y .
\end{gathered}
$$

One should choose a version of $\varphi$ satisfying $\varphi^{\prime}(y)>0, y \in I$. The function $\varphi$ kills the drift $f$ of $\left(x_{t} ; t \geq 0\right)$ in the sense that considering the change of variable $y_{t}=\varphi\left(x_{t}\right)$,

$$
d y_{t}=\left(\varphi^{\prime} g\right)\left(\varphi^{-1}\left(y_{t}\right)\right) d w_{t}, \quad y_{0}=\varphi(x)
$$

The driftless diffusion $\left(y_{t} ; t \geq 0\right)$ is often termed the diffusion in natural coordinates with state-space $[\varphi(0), \varphi(1)]$. Its volatility is $\widetilde{g}(y):=\left(\varphi^{\prime} g\right)\left(\varphi^{-1}(y)\right)$. The function $\varphi$ is often called the scale function.

Whenever $\varphi(0)>-\infty$ and $\varphi(1)<+\infty$, one can choose the integration constants defining $\varphi(x)$ so that

$$
\varphi(x)=\frac{\int_{0}^{x} e^{-2 \int_{0}^{y}\left(f(z) /\left(g^{2}(z)\right)\right) d z} d y}{\int_{0}^{1} e^{-2 \int_{0}^{y}\left(f(z) /\left(g^{2}(z)\right)\right) d z} d y},
$$

with $\varphi(0)=0$ and $\varphi(1)=1$. In this case, the state-space of $\left(y_{t} ; t \geq 0\right)$ is again $[0,1]$, the same as for $\left(x_{t} ; t \geq 0\right)$.

Finally, considering the random time change $t \rightarrow \theta_{t}$ with inverse: $\theta \rightarrow t_{\theta}$ defined by $\theta_{t_{\theta}}=\theta$ and

$$
\theta=\int_{0}^{t_{\theta}} \widetilde{g}^{2}\left(y_{s}\right) d s,
$$


the novel diffusion $\left(w_{\theta}:=y_{t_{\theta}} ; \theta \geq 0\right)$ is easily checked to be identical in law to a standard Brownian motion. Let now $\delta_{y}(\cdot)=$ weak- $\lim _{\varepsilon \downarrow 0}(1 / 2 \varepsilon) \mathbf{1}(\cdot \in(y-\varepsilon, y+\varepsilon))$ stand for the Dirac delta mass at $y$. The random time $\theta_{t}$ can be expressed as

$$
\theta_{t}=\int_{0}^{1} d x \cdot m(x) \int_{0}^{t} \delta_{\varphi(x)}\left(w_{s}\right) d s=\int_{0}^{t} m\left(\varphi^{-1}\left(w_{s}\right)\right) d s,
$$

where $m(x):=1 /\left(g^{2} \varphi^{\prime}\right)(x)$ is the (positive) speed density at $x=\varphi^{-1}(y)$ and $L_{t}(y):=$ $\lim _{\varepsilon \downarrow 0}(1 / 2 \varepsilon) \int_{0}^{t} \mathbf{1}\left(w_{s} \in(y-\varepsilon, y+\varepsilon)\right) d s$ the local time at $y$ of the Brownian motion before time $t$. Both the scale function $\varphi$ and the speed measure $d \mu=m(x) \cdot d x$ are, therefore, essential ingredients to reduce the original stochastic process $\left(x_{t} ; t \geq 0\right)$ to the standard Brownian motion $\left(w_{t} ; t \geq 0\right)$. Indeed, it follows from the above arguments that if $\theta_{t}=\int_{0}^{t} m\left(x_{s}\right) d s$, then $\left(\varphi\left(x_{\theta_{t}}\right) ; t \geq 0\right)$ is a Brownian motion. The Kolmogorov backward infinitesimal generator $G$ may be written in Feller form

$$
G(\cdot)=\frac{1}{2} \frac{d}{d \mu}\left(\frac{d}{d \varphi} \cdot\right)
$$

Examples (from population genetics). (i) Assume that $f(x)=0$ and $g^{2}(x)=x(1-x)$. This is the neutral Wright-Fisher (WF) model discussed at length later. This diffusion is already in natural scale and $\varphi(x)=x, m(x)=[x(1-x)]^{-1}$. The speed measure is not integrable.

(ii) With $u_{1}, u_{2}>0$, assume $f(x)=u_{1}-\left(u_{1}+u_{2}\right) x$ and $g^{2}(x)=x(1-x)$. This is the Wright-Fisher model with mutation. The parameters $u_{1}, u_{2}$ can be interpreted as mutation rates. The drift vanishes when $x=u_{1} /\left(u_{1}+u_{2}\right)$ which is an attracting point for the dynamics. Here, $\varphi^{\prime}(y)=\varphi^{\prime}\left(y_{0}\right) y^{-2 u_{1}}(1-y)^{-2 u_{2}}, \varphi(x)=\varphi\left(x_{0}\right)+\varphi^{\prime}\left(y_{0}\right) \int_{x_{0}}^{x} y^{-2 u_{1}}(1-y)^{-2 u_{2}} d y$, with $\varphi(0)=$ $-\infty$ and $\varphi(1)=+\infty$ if $u_{1}, u_{2}>1 / 2$. The speed measure density is $m(x) \propto x^{2 u_{1}-1}(1-x)^{2 u_{2}-1}$ and so is always integrable.

(iii) With $\sigma \in \mathbf{R}$, assume a model with quadratic logistic drift $f(x)=\sigma x(1-x)$ and local variance $g^{2}(x)=x(1-x)$. This is the WF model with selection. For this diffusion (see [15]), $\varphi(x)=\left(\left(1-e^{-2 \sigma x}\right) /\left(1-e^{-2 \sigma}\right)\right)$ and $m(x) \propto[x(1-x)]^{-1} e^{2 \sigma x}$ are not integrable. Here, $\sigma$ is a selection or fitness parameter. We shall return at length to this model and its neutral version later.

\subsection{The Transition Probability Density}

Assume that $f(x)$ and $g(x)$ are now differentiable in $I$. Let then $p(x ; t, y)$ stand for the transition probability density function of $x_{t}$ at $y$ given $x_{0}=x$. Then, $p:=p(x ; t, y)$ is the smallest solution to the Kolmogorov forward (Fokker-Planck) equation (KFE)

$$
\partial_{t} p=G^{*}(p), \quad p(x ; 0, y)=\delta_{y}(x),
$$

where $G^{*}(\cdot)=-\partial_{y}(f(y) \cdot)+(1 / 2) \partial_{y}^{2}\left(g^{2}(y) \cdot\right)$ is the adjoint of $G\left(G^{*}\right.$ acts on the terminal value $y$, whereas $G$ acts on the initial value $x$ ). The way one can view this PDE depends on the type of boundaries that $\{0,1\}$ are.

We will next suppose that the boundaries $\circ:=0$ or 1 are both exit (or absorbing) boundaries. From the Feller classification of boundaries, this will be the case if for all $y_{0} \in$ $(0,1)$

$$
\text { (i) } m(y) \notin L_{1}\left(y_{0}, 0\right), \quad \text { (ii) } \varphi^{\prime}(y) \int_{y_{0}}^{y} m(z) d z \in L_{1}\left(y_{0}, 0\right),
$$

where a function $f(y) \in L_{1}\left(y_{0}, \circ\right)$ if $\int_{y_{0}}^{\circ}|f(y)| d y<+\infty$. 
In this case, a sample path of $\left(x_{t} ; t \geq 0\right)$ can reach $\circ$ from the inside of $I$ in finite time but cannot reenter. The sample paths are absorbed at 0 . There is an absorption at $\circ$ at time $\tau_{x, 0}=\inf \left(t>0: x_{t}=\circ \mid x_{0}=x\right)$ and $\mathbf{P}\left(\tau_{x, o}<\infty\right)=1$. Whenever both boundaries $\{0,1\}$ are absorbing, the diffusion $x_{t}$ should be stopped at $\tau_{x}:=\tau_{x, 0} \wedge \tau_{x, 1}$. Would none of the boundaries $\{0,1\}$ be absorbing, then $\tau_{x}=+\infty$, which we rule out.

Examples of diffusion with exit boundaries are WF model and WF model with selection. In the WF model including mutations, the boundaries are entrance boundaries and so are not absorbing.

When the boundaries are absorbing, $p(x ; t, y)$ is a subprobability. Letting $\rho_{t}(x):=$ $\int_{0}^{1} p(x ; t, y) d y$, we clearly have $\rho_{t}(x)=\mathbf{P}\left(\tau_{x}>t\right)$. Such models are nonconservative.

For one-dimensional diffusions, the transition density $p(x ; t, y)$ is reversible with respect to the speed density ([8, Chapter 15 , Section 13]) and so detailed balance holds

$$
m(x) p(x ; t, y)=m(y) p(y ; t, x), \quad 0<x, y<1 .
$$

The speed density $m(y)$ satisfies $G^{*}(m)=0$. It may be written as a Gibbs measure with density: $m(y) \propto\left(1 / g^{2}(y)\right) e^{-U(y)}$, where the potential function $U(y)$ reads

$$
U(y):=-2 \int_{0}^{y} \frac{f(z)}{g^{2}(z)} d z, \quad 0<y<1
$$

and with the measure $d y / g^{2}(y)$ standing for the reference measure.

Further, if $p(s, x ; t, y)$ is the transition probability density from $(s, x)$ to $(t, y), s<t$, then $-\partial_{s} p=G(p)$, with terminal condition $p(t, x ; t, y)=\delta_{y}(x)$ and so $p(s, x ; t, y)$ also satisfies the KBE when looking at it backward in time. The Feller evolution semigroup being time homogeneous, one may as well observe that with $p:=p(x ; t, y)$, operating the time substitution $t-s \rightarrow t, p$ itself solves the KBE

$$
\partial_{t} p=G(p), \quad p(x ; 0, y)=\delta_{y}(x) .
$$

In particular, integrating over $y, \partial_{t} \rho_{t}(x)=G\left(\rho_{t}(x)\right)$, with $\rho_{0}(x)=\mathbf{1}(x \in(0,1))$.

$p(x ; t, y)$ being a sub-probability, we may define the normalized conditional probability density $q(x ; t, y):=p(x ; t, y) / \rho_{t}(x)$, now with total mass 1 . We get

$$
\partial_{t} q=-\frac{\partial_{t} \rho_{t}(x)}{\rho_{t}(x)} \cdot q+G^{*}(q), \quad q(x ; 0, y)=\delta_{y}(x) .
$$

The term $b_{t}(x):=-\partial_{t} \rho_{t}(x) / \rho_{t}(x)>0$ is the time-dependent birth rate at which mass should be created to compensate the loss of mass of the original process due to absorption of $\left(x_{t} ; t \geq 0\right)$ at the boundaries. In this creation of mass process, a diffusing particle started in $x$ dies at rate $b_{t}(x)$ at point $(t, y)$, where it is duplicated in two new independent particles both started at $y$ (resulting in a global birth) evolving in the same diffusive way (consider a diffusion process with forward infinitesimal generator $G^{*}$ governing the evolution of $p(x ; t, y)$. Suppose that a sample path of this process has some probability that it will be killed or create a new copy of itself and that the killing and birth rates $d$ and $b$ depend on the current location $y$ of the path. Then, the process with the birth and death opportunities of a path has the infinitesimal 
generator $\lambda(y) \cdot+G^{*}(\cdot)$, where $\lambda(y)=b(y)-d(y)$. The rate can also depend on $t$ and $\left.x\right)$. The birth rate function $b_{t}(x)$ depends here on $x$ and $t$, not on $y$.

When the boundaries of $x_{t}$ are absorbing, the spectra of both $-G$ and $-G^{*}$ are discrete (see [8, Page 330]): There exist positive eigenvalues $\left(\lambda_{k}\right)_{k \geq 1}$ ordered in ascending sizes and eigenvectors $\left(v_{k}, u_{k}\right)_{k \geq 1}$ of both $-G^{*}$ and $-G$ satisfying $-G^{*}\left(v_{k}\right)=\lambda_{k} v_{k}$ and $-G\left(y_{k}\right)=\lambda_{k} u_{k}$ such that with $\left\langle u_{k}, v_{k}\right\rangle:=\int_{0}^{1} u_{k}(x) v_{k}(x) d x$ and $b_{k}:=\left\langle u_{k}, v_{k}\right\rangle^{-1}$, the spectral representation

$$
p(x ; t, y)=\sum_{k \geq 1} b_{k} e^{-\lambda_{k} t} u_{k}(x) v_{k}(y)
$$

holds.

Let $\lambda_{1}>\lambda_{0}=0$ be the smallest nonnull eigenvalue of the infinitesimal generator $-G^{*}$ (and of $-G$ ). Clearly, $-(1 / t) \log \rho_{t}(x) \underset{t \rightarrow \infty}{\rightarrow} \lambda_{1}$ and by L' Hospital rule, therefore, $b_{t}(x) \underset{t \rightarrow \infty}{\rightarrow} \lambda_{1}$. Putting $\partial_{t} q=0$ in the latter evolution equation, independently of the initial condition $x$

$$
q(x ; t, y) \underset{t \rightarrow \infty}{\longrightarrow} q_{\infty}(y)=v_{1}(y)
$$

where $v_{1}$ is the eigenvector of $-G^{*}$ associated to $\lambda_{1}$, satisfying $-G^{*} v_{1}=\lambda_{1} v_{1}$. The limiting probability $v_{1} /$ norm (after a proper normalization) is called the Yaglom limit law of $\left(x_{t} ; t \geq 0\right)$ conditioned on being currently alive at all time $t$ (see [7]).

\subsection{Additive Functionals Along Sample Paths}

Let $\left(x_{t} ; t \geq 0\right)$ be the diffusion model defined by (2.1) on the interval $I$, where both endpoints are assumed absorbing (exit). This process is, thus, transient and nonconservative. We wish to evaluate the nonnegative additive quantities

$$
\alpha(x)=\mathbf{E}\left(\int_{0}^{\tau_{x}} c\left(x_{s}\right) d s+d\left(x_{\tau_{x}}\right)\right)
$$

where the functions $c$ and $d$ are both assumed nonnegative on $I$ and $\partial I=\{0,1\}$. The functional $\alpha(x) \geq 0$ solves the Dirichlet problem

$$
\begin{gathered}
-G(\alpha)=c \quad \text { if } x \in I, \\
\alpha=d \quad \text { if } x \in \partial I,
\end{gathered}
$$

and $\alpha$ is a superharmonic function for $G$, satisfying $-G(\alpha) \geq 0$.

Some Examples.

(1) Assume that $c=1$ and $d=0:$ here, $\alpha=\mathbf{E}\left(\tau_{x}\right)$ is the mean time of absorption (average time spent in $(0,1)$ before absorption), solution to

$$
\begin{gathered}
-G(\alpha)=1 \quad \text { if } x \in I, \\
\alpha=0 \quad \text { if } x \in \partial I .
\end{gathered}
$$


(2) Whenever both $\{0,1\}$ are exit boundaries, it is of interest to evaluate the probability that $x_{t}$ first hits $[0,1]$ (say) at 1 , given $x_{0}=x$. This can be obtained by choosing $c=0$ and $d(0)=\mathbf{1}(0=1)$.

Let then $\alpha=: \alpha_{1}(x)=\mathbf{P}\left(x_{t}\right.$ first hits $[0,1]$ at $\left.1 \mid x_{0}=x\right) . \alpha_{1}(x)$ is a G-harmonic function solution to $G\left(\alpha_{1}\right)=0$, with boundary conditions $\alpha_{1}(0)=0$ and $\alpha_{1}(1)=1$. Solving this problem, we get

$$
\alpha_{1}(x)=\frac{\varphi(x)-\varphi(0)}{\varphi(1)-\varphi(0)}=\frac{\int_{0}^{x} d y e^{-2 \int_{0}^{y}\left(f(z) /\left(g^{2}(z)\right)\right) d z}}{\int_{0}^{1} d y e^{-2 \int_{0}^{y}\left(f(z) /\left(g^{2}(z)\right)\right) d z}} .
$$

On the contrary, choosing $\alpha_{0}(x)$ to be a $G$-harmonic function with boundary conditions $\alpha_{0}(0)=1$ and $\alpha_{0}(1)=0, \alpha_{0}(x)=\mathbf{P}\left(x_{t}\right.$ first hits $[0,1]$ at $\left.0 \mid x_{0}=x\right)=1-\alpha_{1}(x)$.

(3) Let $y \in I$ and put $c=(1 / 2 \varepsilon) \mathbf{1}(x \in(y-\varepsilon, y+\varepsilon))$ and $d=0$. As $\varepsilon \rightarrow 0, c$ converges weakly to $\delta_{y}(x)$ and, $\alpha=: \mathfrak{g}(x, y)=\mathbf{E}\left(\lim (1 / 2 \varepsilon) \int_{0}^{\tau_{x}} \mathbf{1}\left(x_{s} \in(y-\varepsilon, y+\varepsilon)\right) d s\right)=\int_{0}^{\infty} p(x ; s, y) d s$ is the Green function, solution to

$$
\begin{gathered}
-G(\mathfrak{g})=\delta_{y}(x) \quad \text { if } x \in I, \\
\mathfrak{g}=0 \quad \text { if } x \in \partial I .
\end{gathered}
$$

$\mathfrak{g}$ is, therefore, the mathematical expectation of the local time at $y$, starting from $x$ (the sojourn time density at $y$ ). The solution is known to be (see [8, page 198] or [5, page 280])

$$
\begin{aligned}
& \mathfrak{g}(x, y)=2 \frac{(\varphi(x)-\varphi(0))(\varphi(1)-\varphi(y))}{\left(g^{2} \varphi^{\prime}\right)(y)(\varphi(1)-\varphi(0))} \quad \text { if } x \leq y, \\
& \mathfrak{g}(x, y)=2 \frac{(\varphi(1)-\varphi(x))(\varphi(y)-\varphi(0))}{\left(g^{2} \varphi^{\prime}\right)(y)(\varphi(1)-\varphi(0))} \quad \text { if } x \geq y .
\end{aligned}
$$

The Green function is of particular interest to solve the general problem of evaluating additive functionals $\alpha(x)$. Indeed, as is well known, see [8], for example, the integral operator with respect to the Green kernel inverts the second-order operator $-G$ leading to

$$
\begin{gathered}
\alpha(x)=\int_{I} \mathfrak{g}(x, y) c(y) d y \quad \text { if } x \in I, \\
\alpha=d \quad \text { if } x \in \partial I .
\end{gathered}
$$

Under this form, $\alpha(x)$ appears as a potential function and all potential function is superharmonic. Note that for all harmonic function $h \geq 0$ satisfying $-G(h)=0$,

$$
\alpha_{h}(x):=\int_{I} \mathfrak{g}(x, y) c(y) d y+h(x)
$$

is again superharmonic because $-G\left(\alpha_{h}\right)=c \geq 0$. 
(4) Also of interest are the additive functionals of the type

$$
\alpha_{\lambda}(x)=\mathbf{E}\left(\int_{0}^{\tau_{x}} e^{-\lambda s} c\left(x_{s}\right) d s+d\left(x_{\tau_{x}}\right)\right)
$$

where the functions $c$ and $d$ are again both assumed to be nonnegative. The functional $\alpha_{\lambda}(x) \geq$ 0 solves the Dynkin problem, [8]

$$
\begin{gathered}
(\lambda I-G)\left(\alpha_{\curlywedge}\right)=c \quad \text { if } x \in I, \\
\alpha_{\lambda}=d \quad \text { if } x \in \partial I
\end{gathered}
$$

involving the action of the resolvent operator $(\lambda I-G)^{-1}$ on $c$.

Whenever $c(x)=\delta_{y}(x), d=0$, then

$$
\alpha_{\lambda}=: \mathfrak{g}_{\lambda}(x, y)=\mathbf{E}\left(\int_{0}^{\tau_{x}} e^{-\lambda s} \delta_{y}\left(x_{s}\right) d s\right)=\int_{0}^{\infty} e^{-\lambda s} p(x ; s, y) d s
$$

is the $\lambda$-potential function, solution to

$$
\begin{gathered}
(\lambda I-G)\left(\mathfrak{g}_{\lambda}\right)=\delta_{y}(x) \quad \text { if } x \in I, \\
\mathfrak{g}_{\lambda}=0 \quad \text { if } x \in \partial I .
\end{gathered}
$$

$\mathfrak{g}_{\mathfrak{l}}$ is, therefore, the mathematical expectation of the exponentially damped local time at $y$, starting from $x$ (the temporal Laplace transform of the transition probability density from $x$ to $y$ at $t$ ), with $g_{0}=g$. Then, it holds that

$$
\begin{gathered}
\alpha_{\lambda}(x)=\int_{I} \mathfrak{g}_{\lambda}(x, y) c(y) d y \quad \text { if } x \in I \\
\alpha_{\curlywedge}=d \quad \text { if } x \in \partial I .
\end{gathered}
$$

The $\lambda$-potential function is also useful in the computation of the distribution of the firstpassage time $\tau_{x, y}$ to $y$ starting from $x$. From the convolution formula,

$$
p(x ; t, y)=\int_{0}^{t} \mathbf{P}\left(\tau_{x, y} \in d s\right) p(y ; t-s, y)
$$

and taking the Laplace transform of both sides with respect to time, we obtain the LaplaceStieltjes transform (LST) of the law of $\tau_{x, y}$ as

$$
\mathbf{E}\left(e^{-\lambda \tau_{x, y}}\right)=\frac{\mathfrak{g}_{\lambda}(x, y)}{\mathfrak{g}_{\lambda}(y, y)}
$$


We have $\mathbf{P}\left(\tau_{x, y}<\infty\right)=\mathfrak{g}(x, y) /(\mathfrak{g}(y, y)) \in(0,1)$ as a result of both terms in the ratio being finite and $x, y$ belonging to the same transience class of the process (under our assumptions that the boundaries are absorbing). Note that from the reversibility property

$$
m(x) \mathfrak{g}(x, y)=m(y) \mathfrak{g}(y, x) .
$$

\subsection{Transformation of Sample Paths (Doob-Transform) and Killing}

In the preceding subsections, we have dealt with a given process and recalled the various ingredients for the expectations of various quantities of interest, summing over the history of paths. In this setup, there is no distinction among paths with different destinations nor did we allow for annihilation or creation of paths inside the domain before the process reached one of the boundaries. The Doob transform of paths allows to do so.

Consider a one-dimensional diffusion $\left(x_{t} ; t \geq 0\right)$ as in (2.1) with absorbing barriers. Let $p(x ; t, y)$ be its transition probability, and let $\tau_{x}$ be its absorption time at the boundaries.

Let $\alpha(x):=\mathbf{E}\left(\int_{0}^{\tau_{x}} c\left(x_{s}\right) d s+d\left(x_{\tau_{x}}\right)\right)$ be a nonnegative additive functional solving

$$
\begin{gathered}
-G(\alpha)=c \quad \text { if } x \in I, \\
\alpha=d \quad \text { if } x \in \partial I .
\end{gathered}
$$

Recall the functions $c$ and $d$ are both chosen nonnegative so that so is $\alpha$.

Define a new transformed stochastic process $\left(\bar{x}_{t} ; t \geq 0\right)$ by its transition probability

$$
\bar{p}(x ; t, y)=\frac{\alpha(y)}{\alpha(x)} p(x ; t, y) .
$$

In this construction of $\left(\bar{x}_{t} ; t \geq 0\right)$ through a change of measure, sample paths of $\left(x_{t} ; t \geq 0\right)$ for which $\alpha(y)$ is large are favored. This is a selection of paths procedure due to Doob (see [11]).

Now, the KFE for $\bar{p}$ clearly is $\partial_{t} \bar{p}=\bar{G}^{*}(\bar{p})$, with $p(x ; 0, y)=\delta_{y}(x)$ and $\bar{G}^{*}(\bar{p})=$ $\alpha(y) G^{*}(\bar{p} / \alpha(y))$. The Kolmogorov backward operator of the transformed process is, therefore, by duality

$$
\bar{G}(\cdot)=\frac{1}{\alpha(x)} G(\alpha(x) \cdot)
$$

Developing, with $\alpha^{\prime}(x):=d \alpha(x) / d x$ and $\widetilde{G}(\cdot):=\left(\alpha^{\prime} / \alpha\right) g^{2} \partial_{x}(\cdot)+G(\cdot)$, we get

$$
\bar{G}(\cdot)=\frac{1}{\alpha} G(\alpha) \cdot+\tilde{G}(\cdot)=-\frac{c}{\alpha} \cdot+\tilde{G}(\cdot),
$$

and the new KB operator can be obtained from the latter by adding a drift term $\left(\alpha^{\prime} / \alpha\right) g^{2} \partial_{x}$ to the one in $G$ of the original process to form a new process $\left(\tilde{x}_{t} ; t \geq 0\right)$ with the KB operator $\tilde{G}$ and by killing its sample paths at death rate $d(x):=(c / \alpha)(x)$ (provided $c \neq 0)$. Note that

$$
d(x)=\frac{1}{(1 /(c(x))) \int_{0}^{1} \mathfrak{g}(x, y) c(y) d y}
$$


In others words, with $\tilde{f}(x):=f(x)+\left(\alpha^{\prime} / \alpha\right) g^{2}(x)$, the novel time-homogeneous SDE to consider is

$$
d \tilde{x}_{t}=\tilde{f}\left(\tilde{x}_{t}\right) d t+g\left(\tilde{x}_{t}\right) d w_{t}, \quad \tilde{x}_{0}=x \in(0,1),
$$

possibly killed at rate $d=c / \alpha$ as soon as $c \neq 0$. Whenever $\left(\tilde{x}_{t} ; t \geq 0\right)$ is killed, it enters conventionally into some coffin state $\{\partial\}$ added to the state-space. Let $\tilde{\tau}_{x}$ be the new absorption time at the boundaries of $\left(\tilde{x}_{t} ; t \geq 0\right)$ started at $x$ (with $\tilde{\tau}_{x}=\infty$ would the boundaries be inaccessible to the new process $\tilde{x}_{t}$ which we ruled out). Let $\tilde{\tau}_{x, \partial}$ be the killing time of $\left(\tilde{x}_{t} ; t \geq 0\right)$ started at $x$ (the hitting time of $\partial$ ), with $\tilde{\tau}_{x, \partial}=\infty$ if $c=0$. Then, $\bar{\tau}_{x}:=\tilde{\tau}_{x} \wedge \widetilde{\tau}_{x, \partial}$ is the novel stopping time of $\left(\tilde{x}_{t} ; t \geq 0\right)$. The SDE for $\left(\tilde{x}_{t} ; t \geq 0\right)$, together with its global stopping time $\bar{\tau}_{x}$ characterize the new process $\left(\bar{x}_{t} ; t \geq 0\right)$ with generator $\bar{G}$ to consider.

In the sequel, we shall limit ourselves to the cases for which the following additional conditions hold on the transformed process.

\section{(i) Nonconservativeness of $\tilde{x}_{t}$.}

We will next suppose that the boundaries $\circ:=0$ or 1 are both exit (or absorbing) boundaries for the new process $\tilde{x}_{t}$ in (2.38). From the Feller criterion for exit boundaries, this will be the case if for all $y_{0} \in(0,1)$

$$
\tilde{m}(y) \notin L_{1}\left(y_{0}, o\right), \quad \tilde{\varphi}^{\prime}(y) \int_{y_{0}}^{y} \tilde{m}(z) d z \in L_{1}\left(y_{0}, o\right),
$$

where $\tilde{m}(y)=1 /\left(\left(g^{2} \tilde{\varphi}^{\prime}\right)(y)\right)$ is the new speed measure density for $\tilde{x}_{t}$ and $\tilde{\varphi}$ its scale function. Recalling $\tilde{f}=f+\left(\alpha^{\prime} / \alpha\right) g^{2}$ and $\tilde{g}^{2}=g^{2}$, we have

$$
\begin{gathered}
\tilde{\varphi}^{\prime}(y)=\tilde{\varphi}^{\prime}\left(y_{0}\right) e^{-2 \int_{y_{0}}^{y}\left(\tilde{f}(z) /\left(g^{2}(z)\right)\right) d z}=\frac{\tilde{\varphi}^{\prime}\left(y_{0}\right)}{\alpha^{2}(y)} \varphi^{\prime}(y), \\
\tilde{\varphi}(x)=\tilde{\varphi}\left(x_{0}\right)+\tilde{\varphi}^{\prime}\left(y_{0}\right) \int_{x_{0}}^{x} \alpha^{-2}(y) e^{-2 \int_{y_{0}}^{y}\left(f(z) /\left(g^{2}(z)\right)\right) d z} d y .
\end{gathered}
$$

So, we assume here that $\tilde{x}_{t}$ obeys itself a nonconservative diffusion.

\section{(ii) Boundedness of the Killing Rate d.}

In some examples, the killing rate $d=-G(\alpha) / \alpha$ is bounded above. For example, suppose that the drift of the diffusion process $\left(x_{t} ; t \geq 0\right)$ is bounded above by $f_{*}=\max _{x}(f(x))>0$. (If the drift of $\left(x_{t} ; t \geq 0\right)$ is bounded below by $f_{*}<0$, we are led to the same conclusions while considering the process $1-x_{t}$ instead of $x_{t}$.) Then, choosing $\alpha(x)=e^{-a x}, a>0,-G(\alpha)=$ $\left(a f-\left(a^{2} / 2\right) g^{2}\right) \alpha<a f_{*} \alpha$. Thus, $d=-G(\alpha) / \alpha$ is bounded above by $a f_{*}$. Because $-G(\alpha)=c \geq 0$, all this makes sense if, for all $x, a f(x)-\left(a^{2} / 2\right) g^{2}(x) \geq 0$ or $-\partial U:=2 f / g^{2} \geq a$ (the opposite of the gradient of the potential function $U$ in (2.12) is bounded below). 
Let $\left(a_{k} ; k \geq 1\right)$ be a nonincreasing sequence of $[0,1]$-valued real numbers. Let $\left(\alpha_{k} ; k \geq 1\right)$ be a sequence of nonnegative real numbers such that for all $x \in(0,1)$

$$
\alpha(x)=\sum_{k \geq 1} \alpha_{k} e^{-a_{k} x}<\infty
$$

Whenever $f$ is bounded above and, for all $x, 2 f / g^{2} \geq a_{1}$, we have

$$
\begin{aligned}
-G(\alpha) & =\sum_{k \geq 1} \alpha_{k}\left(a_{k} f-\frac{a_{k}^{2}}{2} g^{2}\right) e^{-a_{k} x} \\
& \leq f_{*} \sum_{k \geq 1} \alpha_{k} a_{k} e^{-a_{k} x}<a_{1} f_{*} \sum_{k \geq 1} \alpha_{k} e^{-a_{k} x}=a_{1} f_{*} \alpha .
\end{aligned}
$$

Thus, $d=-G(\alpha) / \alpha$ is bounded above by $a_{1} f_{*}$.

Therefore, for a large class of diffusion processes, the exponential function or some linear combinations of exponential functions are superharmonic functions $\alpha$, leading to a bounded above killing rate $d=-G(\alpha) / \alpha$.

\subsection{Normalizing and Conditioning}

Because the transformed process $\bar{x}_{t}$ is nonconservative, it is of interest to inspect various conditionings in the sense of Yaglom, [7].

(i) Consider again the process with infinitesimal generator $\bar{G}$ losing mass due to killing and/or absorption at the boundaries. Integrating over $y$, with $\bar{\rho}_{t}(x):=\int_{I} \bar{p}(x ; t, y) d y=\mathbf{P}\left(\bar{\tau}_{x}>\right.$ $t$ ), we have

$$
\partial_{t} \bar{\rho}_{t}(x)=\bar{G}\left(\bar{\rho}_{t}(x)\right)=-d(x) \bar{\rho}_{t}(x)+\widetilde{G}\left(\bar{\rho}_{t}(x)\right),
$$

with $\bar{\rho}_{0}(x)=\mathbf{1}(x \in(0,1))$. This gives the tail distribution of the full stopping time $\bar{\tau}_{x}$.

Defining the conditional probability density $\bar{q}(x ; t, y):=\bar{p}(x ; t, y) / \bar{\rho}_{t}(x)$, now with total mass 1 , with $\bar{q}(x ; 0, y)=\delta_{y}(x)$, we get

$$
\begin{aligned}
\partial_{t} \bar{q} & =-\frac{\partial_{t} \bar{\rho}_{t}(x)}{\bar{\rho}_{t}(x)} \cdot \bar{q}+\bar{G}^{*}(\bar{q}) \\
& =\left(\bar{b}_{t}(x)-d(y)\right) \cdot \bar{q}+\widetilde{G}^{*}(\bar{q}) .
\end{aligned}
$$

The term $\bar{b}_{t}(x)=-\partial_{t} \bar{\rho}_{t}(x) / \bar{\rho}_{t}(x)>0$ is the rate at which mass should be created to compensate the loss of mass of the process $\left(\tilde{x}_{t} ; t \geq 0\right)$ due to its possible absorption at the boundaries and/or killing. Again, we have $\bar{b}_{t}(x) \rightarrow \lambda_{1}$, where $\lambda_{1}$ is the smallest positive eigenvalue of $-G$, and therefore, putting $\partial_{t} \bar{q}=0$ in the latter evolution equation, we get that independently of the initial condition $x$

$$
\bar{q}(x ; t, y) \underset{t \rightarrow \infty}{\longrightarrow} \bar{q}_{\infty}(y)
$$


where $\bar{q}_{\infty}(y)$ is the solution to

$$
\begin{gathered}
-\widetilde{G}^{*}\left(\bar{q}_{\infty}\right)=\left(\lambda_{1}-d(y)\right) \cdot \bar{q}_{\infty} \text { or } \\
-\bar{G}^{*}\left(\bar{q}_{\infty}\right)=\lambda_{1} \cdot \bar{q}_{\infty} .
\end{gathered}
$$

With $v_{1}$ the eigenvector of $-G^{*}$ associated to $\lambda_{1}, \bar{q}_{\infty}(y)$ is of the product form

$$
\bar{q}_{\infty}(y)=\frac{\alpha(y) v_{1}(y)}{\left\langle\alpha, v_{1}\right\rangle}
$$

where $\left\langle\alpha, v_{1}\right\rangle=\int_{0}^{1} \alpha(y) v_{1}(y) d y$. This results directly from the fact that $\bar{G}^{*}\left({ }^{\circ}\right)=\alpha(y) G^{*}(\cdot / \alpha(y))$ and that $v_{1}$ is the stated eigenvector of $-G^{*}$. A different way to see this is as follows. We have

$$
\bar{\rho}_{t}(x)=\frac{1}{\alpha(x)} \int_{0}^{1} \alpha(y) p(x ; t, y) d y
$$

and the conditional density of $\bar{x}_{t}$ given $\bar{\tau}_{x}>t$ is, therefore,

$$
\bar{q}(x ; t, y)=\frac{\alpha(y) p(x ; t, y)}{\int_{0}^{1} \alpha(y) p(x ; t, y) d y} .
$$

The rest follows from observing that, to the leading order in $t$ in (2.15), for large time

$$
p(x ; t, y) \sim b_{1} e^{-\lambda_{1} t} \cdot u_{1}(x) v_{1}(y)
$$

where $u_{1}\left(v_{1}\right.$, resp. $)$ is the eigenvector of $-G\left(-G^{*}\right.$, resp.) associated to $\lambda_{1}$ and $b_{1}=\left\langle u_{1}, v_{1}\right\rangle^{-1}$. From this, it is clear that $-(1 / t) \log \bar{\rho}_{t}(x) \underset{t \rightarrow \infty}{\rightarrow} \lambda_{1}$ and

$$
\bar{q}(x ; t, y) \sim \frac{e^{-\lambda_{1} t} \cdot \alpha(y) v_{1}(y)}{e^{-\lambda_{1} t} \cdot\left\langle\alpha, v_{1}\right\rangle}=\bar{q}_{\infty}(y)
$$

The limiting probability $\bar{q}_{\infty}=\alpha v_{1} /$ norm can, therefore, be interpreted as the Yaglom limit law of $\left(\bar{x}_{t} ; t \geq 0\right)$ conditioned on the event $\bar{\tau}_{x}>t$.

(ii) Under our assumptions, in the transformation of paths process, the transformed process $\left(\bar{x}_{t} ; t \geq 0\right)$ can both be absorbed at the boundaries and be killed. So, both $\widetilde{\tau}_{x}$ and $\widetilde{\tau}_{x, \partial}$ are finite with positive probability. We wish to understand the processes $\left(\bar{x}_{t} ; t \geq 0\right)$ conditioned on the events $\left\{\widetilde{\tau}_{x}<\widetilde{\tau}_{x, \partial}\right\}$ or $\left\{\widetilde{\tau}_{x, \partial}<\tilde{\tau}_{x}\right\}$, (see [16]).

The probability mass cumulated at the boundaries $\{0,1\}$ by time $t$ clearly is [17]

$$
\mathbf{P}\left(\tilde{\tau}_{x} \leq t\right)=\mathbf{P}\left(\tilde{\tau}_{x, \partial}>t\right)=\frac{1}{2}\left(\int_{0}^{t}[\bar{p}(x ; s, 0)+\bar{p}(x ; s, 1)] d s\right) .
$$


As $t \rightarrow \infty$, this probability tends to $\mathbf{P}\left(\widetilde{\tau}_{x, \partial}=\infty\right)=\mathbf{P}\left(\tilde{\tau}_{x}<\widetilde{\tau}_{x, \partial}\right)=: \beta(x)$. Note that

$$
\beta(x)=\mathbf{P}\left(\tilde{x}_{\bar{\tau}_{x}} \in\{0,1\}\right)=\mathbf{E}\left(\mathbf{1}\left(\tilde{x}_{\bar{\tau}_{x}} \in\{0,1\}\right)\right) .
$$

Now (assuming $x \neq\{0,1\}$ ),

$$
\begin{aligned}
\bar{G}\left(\mathbf{P}\left(\widetilde{\tau}_{x, \partial}>t\right)\right) & =\frac{1}{2}\left(\int_{0}^{t}[\bar{G}(\bar{p}(x ; s, 0))+\bar{G}(\bar{p}(x ; s, 1))] d s\right) \\
& =\frac{1}{2}\left(\int_{0}^{t}\left[\partial_{s}(\bar{p}(x ; s, 0))+\partial_{s}(\bar{p}(x ; s, 1))\right] d s\right) \\
& =\frac{1}{2}(\bar{p}(x ; t, 0)+\bar{p}(x ; t, 1)) \underset{t \rightarrow \infty}{\longrightarrow} 0 .
\end{aligned}
$$

Thus, $\beta$ is defined by

$$
\bar{G}(\beta(x))=0,
$$

[or $\tilde{G}(\beta(x))=d(x) \beta(x)$ ], with boundary conditions $\beta(0)=\beta(1)=1$. It serves as a positive harmonic function for $\bar{G}$. This is a Sturm-Liouville problem to be solved for each case study.

The density of the process $\left(\bar{x}_{t} ; t \geq 0\right)$ conditioned on the event $\left\{\tilde{\tau}_{x}<\tilde{\tau}_{x, a}\right\}$ is

$$
\bar{p}_{a}(x ; t, y)=\frac{\beta(y)}{\beta(x)} \bar{p}(x ; t, y)=\frac{\beta(y)}{\beta(x)} \frac{\alpha(y)}{\alpha(x)} p(x ; t, y) .
$$

The density of the process $\left(\bar{x}_{t} ; t \geq 0\right)$ conditioned on the event $\left\{\tilde{\tau}_{x, \partial}<\tilde{\tau}_{x}\right\}$ is

$$
\bar{p}_{\partial}(x ; t, y)=\frac{1-\beta(y)}{1-\beta(x)} \bar{p}(x ; t, y)=\frac{1-\beta(y)}{1-\beta(x)} \frac{\alpha(y)}{\alpha(x)} p(x ; t, y)
$$

Note that

$$
\begin{aligned}
& \int_{0}^{1} \bar{p}_{a}(x ; t, y) d y=\mathbf{P}\left(\tilde{t}_{x}>t \mid \tilde{\tau}_{x}<\tilde{\tau}_{x, \partial}\right), \\
& \int_{0}^{1} \bar{p}_{\partial}(x ; t, y) d y=\mathbf{P}\left(\tilde{\tau}_{x, \partial}>t \mid \tilde{\tau}_{x, \partial}<\tilde{\tau}_{x}\right),
\end{aligned}
$$


and, with $\bar{\tau}_{x}=\tilde{\tau}_{x} \wedge \tilde{\tau}_{x, \partial}$,

$$
\begin{aligned}
\mathbf{P}\left(\bar{\tau}_{x}>t\right) & =\beta(x) \mathbf{P}\left(\tilde{\tau}_{x}>t \mid \tilde{\tau}_{x}<\tilde{\tau}_{x, \partial}\right)+(1-\beta(x)) \mathbf{P}\left(\tilde{\tau}_{x, \partial}>t \mid \tilde{\tau}_{x, \partial}<\tilde{\tau}_{x}\right) \\
& =\beta(x) \int_{0}^{1} \frac{\beta(y)}{\beta(x)} \bar{p}(x ; t, y) d y+(1-\beta(x)) \int_{0}^{1} \frac{1-\beta(y)}{1-\beta(x)} \bar{p}(x ; t, y) d y \\
& =\int_{0}^{1} \bar{p}(x ; t, y) d y,
\end{aligned}
$$

as required, because this is the probability that $\bar{x}$. is neither in $\{0,1\}$ nor in state $\partial$ at time $t$.

Note also that $\bar{q}_{a}(x ; t, y)=\bar{p}_{a}(x ; t, y) / \int_{0}^{1} \bar{p}_{a}(x ; t, y) d y\left[\operatorname{resp} ., \bar{q}_{\partial}(x ; t, y)=\bar{p}_{\partial}(x ; t, y) /\right.$ $\left.\int_{0}^{1} \bar{p}_{\partial}(x ; t, y) d y\right]$ are the transition probability densities of $\left(\bar{x}_{t} ; t \geq 0\right)$ conditioned on the event $\left\{\widetilde{\tau}_{x}<\widetilde{\tau}_{x, \partial}\right.$ and $\left.\widetilde{\tau}_{x}>t\right\}$ [of $\left(\bar{x}_{t} ; t \geq 0\right)$ conditioned on the event $\left\{\widetilde{\tau}_{x, \partial}<\widetilde{\tau}_{x}\right.$ and $\widetilde{\tau}_{x, \partial}>t$, resp. $\}$ ]. They are the Yaglom limits of both conditioned processes.

The backward infinitesimal generators of both processes with transition probability densities $\bar{p}_{a}$ and $\bar{p}_{\partial}$ are, respectively, given by

$$
\begin{gathered}
\bar{G}_{a}(\cdot)=\frac{1}{\beta(x)} \bar{G}(\beta(x) \cdot), \\
\bar{G}_{\partial}(\cdot)=\frac{1}{1-\beta(x)} \bar{G}((1-\beta(x)) \cdot) .
\end{gathered}
$$

We get, respectively,

$$
\begin{gathered}
\bar{G}_{a}(\cdot)=\tilde{G}(\cdot)+\frac{\beta^{\prime} g^{2}}{\beta}(x) \partial_{x}(\cdot), \\
\bar{G}_{\partial}(\cdot)=-\frac{d}{1-\beta} \cdot+\tilde{G}(\cdot)-\frac{\beta^{\prime} g^{2}}{1-\beta}(x) \partial_{x}(\cdot) .
\end{gathered}
$$

Thus, in $\bar{G}_{a}(\cdot)$, there is no multiplicative part (no killing) and a shift in the drift, showing that the associated conditioned process $\left(\tilde{x}_{a, t} ; t \geq 0\right)$ obeys the SDE

$$
d \tilde{x}_{a}=\tilde{f}_{a}\left(\tilde{x}_{a}\right) d t+g\left(\tilde{x}_{a}\right) d w_{t}
$$

with drift

$$
\begin{aligned}
\tilde{f}_{a}(x) & =\tilde{f}(x)+\frac{\beta^{\prime} g^{2}}{\beta}(x)=f(x)+\frac{\alpha^{\prime} g^{2}}{\alpha}(x)+\frac{\beta^{\prime} g^{2}}{\beta}(x) \\
& =f(x)+g^{2}(x)\left[\frac{\alpha^{\prime}}{\alpha}(x)+\frac{\beta^{\prime}}{\beta}(x)\right] .
\end{aligned}
$$

This process is ultimately absorbed at $\{0,1\}$. 
In $\bar{G}_{\partial}(\cdot)$, there is a killing multiplicative part which is enhanced $d /(1-\beta)>d$ and a shift in the drift, showing that the associated conditioned process $\left(\tilde{x}_{\partial, t} ; t \geq 0\right)$ exhibits a faster killing rate, but the drift shift guarantees that $\left(\tilde{x}_{\partial, t} ; t \geq 0\right)$ is not absorbed at the boundaries. We have

$$
\tilde{f}_{\partial}(x)=f(x)+g^{2}(x)\left[\frac{\alpha^{\prime}}{\alpha}(x)-\frac{\beta^{\prime}}{1-\beta}(x)\right]
$$

Additive Functionals of the Transformed Process.

for the new process $\left(\tilde{x}_{t} ; t \geq 0\right)$, it is also of interest to evaluate additive functionals along their own sample paths. Let then $\tilde{\alpha}(x):=\mathrm{E}\left(\int_{0}^{\bar{\tau}_{x}} \widetilde{c}\left(\tilde{x}_{s}\right) d s+\tilde{d}\left(\tilde{x}_{\bar{\tau}_{x}}\right)\right)$ be such an additive functional where the functions $\tilde{c}$ and $\tilde{d}$ are themselves both nonnegative. It solves

$$
\begin{gathered}
-\bar{G}(\widetilde{\alpha})=\tilde{c} \quad \text { if } x \in I, \\
\tilde{\alpha}=\tilde{d} \quad \text { if } x \in \partial I .
\end{gathered}
$$

Then, recalling the expression of the Green function $\mathfrak{g}(x, y)$ of $\left(x_{t} ; t \geq 0\right)$ in $(2.22)$, we find explicitly

$$
\tilde{\alpha}(x)=\frac{1}{\alpha(x)} \int_{I} \mathfrak{g}(x, y) \alpha(y) \tilde{c}(y) d y .
$$

Specific transformations of interest.

(i) The case $c=0$ deserves a special treatment. Indeed, in this case, $\tilde{\tau}_{x, \partial}=\infty$ and so $\bar{\tau}_{x}:=\tilde{\tau}_{x}$, the absorption time for the process $\left(\tilde{x}_{t} ; t \geq 0\right)$ governed by the new SDE (2.38). Here, $\bar{G}=\widetilde{G}$. Assuming $\alpha$ solves $-G(\alpha)=0$ if $x \in I$ with boundary conditions $\alpha(0)=0$ and $\alpha(1)=1$ $(\alpha(0)=1$ and $\alpha(1)=0$, resp. $)$, the new process $\left(\tilde{x}_{t} ; t \geq 0\right)$ is just $\left(x_{t} ; t \geq 0\right)$ conditioned on exiting at $x=1$ (at $x=0$, resp.). In the first case, the boundary 1 is exit, whereas 0 is entrance; $\alpha$ reads

$$
\alpha(x)=\frac{\int_{0}^{x} e^{-2 \int_{0}^{y}\left(f(z) /\left(g^{2}(z)\right)\right) d z} d y}{\int_{0}^{1} e^{-2 \int_{0}^{y}\left(f(z) /\left(g^{2}(z)\right)\right) d z} d y}
$$

with

$$
\tilde{f}(x)=f(x)+\frac{g^{2}(x) e^{-2 \int_{0}^{x}\left(f(z) /\left(g^{2}(z)\right)\right) d z}}{\int_{0}^{x} e^{-2 \int_{0}^{y}\left(f(z) /\left(g^{2}(z)\right)\right) d z} d y}
$$

giving the new drift. In the second case, $\alpha(x)=\left(\int_{x}^{1} e^{-2 \int_{0}^{y}\left(f(z) / g^{2}(z)\right) d z} d y /\left(\int_{0}^{1} e^{-2 \int_{0}^{y}\left(f(z) / g^{2}(z)\right) d z} d y\right)\right)$, and the boundary 0 is exit, whereas 1 is entrance. Thus, $\tilde{\tau}_{x}$ is just the exit time at $x=1$ (at $x=0$, resp.). Let $\widetilde{\alpha}(x):=\mathbf{E}\left(\widetilde{\tau}_{x}\right)$. Then, $\widetilde{\alpha}(x)$ solves $-\widetilde{G}(\widetilde{\alpha})=1$, whose explicit solution is

$$
\tilde{\alpha}(x)=\frac{1}{\alpha(x)} \int_{I} \mathfrak{g}(x, y) \alpha(y) d y
$$

in terms of $\mathfrak{g}(x, y)$, the Green function of $\left(x_{t} ; t \geq 0\right)$. 
Example 2.1. Consider the WF model on [0,1] with selection for which, with $\sigma \in \mathbf{R}, f(x)=$ $\sigma x(1-x)$ and $g^{2}(x)=x(1-x)$. Assume that $\alpha$ solves $-G(\alpha)=0$ if $x \in(0,1)$ with $\alpha(0)=0$ and $\alpha(1)=1$; one gets, $\alpha(x)=\left(1-e^{-2 \sigma x}\right) /\left(1-e^{-2 \sigma}\right)$. The diffusion corresponding to (2.38) has the new drift: $\tilde{f}(x)=\sigma x(1-x) \operatorname{coth}(\sigma x)$, independently of the sign of $\sigma$. It models the WF diffusion with selection conditioned on exit at $\circ=1$.

(ii) Assume that $\alpha$ now solves $-G(\alpha)=1$ if $x \in I$ with boundary conditions $\alpha(0)=$ $\alpha(1)=0$. In this case study, one selects sample paths of $\left(x_{t} ; t \geq 0\right)$ with a large mean absorption time $\alpha(x)=\mathrm{E}\left(\tau_{x}\right)$. Sample paths with large sojourn time in $I$ are favored. We have

$$
\alpha(x)=\int_{I} \mathfrak{g}(x, y) d y,
$$

where $\mathfrak{g}(x, y)$ is the Green function (2.22). The boundaries of $\left(\tilde{x}_{t} ; t \geq 0\right)$ are now both entrance boundaries and so $\tilde{\tau}_{x}=\infty$. $\left(\tilde{x}_{t} ; t \geq 0\right)$ is not absorbed at the boundaries. The stopping time $\bar{\tau}_{x}$ of $\left(\tilde{x}_{t} ; t \geq 0\right)$ is just its killing time $\widetilde{\tau}_{x, \partial}$. Let $\tilde{\alpha}(x):=\mathbf{E}\left(\widetilde{\tau}_{x, \partial}\right)$. Then, $\tilde{\alpha}(x)$ solves $-\bar{G}(\widetilde{\alpha})=1$, $\tilde{\alpha}(0)=\tilde{\alpha}(1)=0$, with explicit solution

$$
\tilde{\alpha}(x)=\frac{1}{\alpha(x)} \int_{I} \mathfrak{g}(x, y) \alpha(y) d y
$$

(iii) Assume that $\alpha$ now solves $-G(\alpha)=\delta_{y}(x)$ if $x \in I$ with boundary conditions $\alpha(0)=\alpha(1)=0$. In this case study, one selects sample paths of $\left(x_{t} ; t \geq 0\right)$ with a large sojourn time density at $y$ recalling $\alpha(x)=: \mathfrak{g}(x, y)=\mathrm{E}\left(\int_{0}^{\tau_{x}} \delta_{y}\left(x_{s}\right) d s\right)$. The stopping time $\tilde{\tau}_{y}(x)$ of $\left(\tilde{x}_{t} ; t \geq 0\right)$ occurs at rate $\delta_{y}(x) / \mathfrak{g}(x, y)$. It is a killing time when the process is at $y$ for the last time after a geometrically distributed number of passages there with rate $1 / \mathfrak{g}(x, y)$ (or with success probability $1 /(1+\mathfrak{g}(x, y)))$. Let $\widetilde{\alpha}_{y}(x):=\mathbf{E}\left(\widetilde{\tau}_{y}(x)\right)$. Then, $\widetilde{\alpha}_{y}(x)$ solves $-\bar{G}(\widetilde{\alpha})=1$, with explicit solution

$$
\tilde{\alpha}_{y}(x)=\frac{1}{\mathfrak{g}(x, y)} \int_{I} \mathfrak{g}(x, z) \mathfrak{g}(z, y) d z .
$$

when $x=1 / N, \tilde{\alpha}_{y}(1 / N)$ may be viewed as the age of a mutant currently observed to present frequency $y$, see [18].

The Green function at $y_{0} \in(0,1)$ of the transformed process $\left(\tilde{x}_{t} ; t \geq 0\right)$ is $\tilde{\mathfrak{g}}_{y}\left(x, y_{0}\right)$ solution to $-\bar{G}\left(\tilde{\mathfrak{g}}_{y}\right)=\delta_{y_{0}}(x)$. It takes the simple form

$$
\tilde{\mathfrak{g}}_{y}\left(x, y_{0}\right)=\frac{1}{\mathfrak{g}(x, y)} \int_{I} \mathfrak{g}(x, z) \mathfrak{g}(z, y) \delta_{y_{0}}(z) d z=\frac{\mathfrak{g}\left(y_{0}, y\right)}{\mathfrak{g}(x, y)} \mathfrak{g}\left(x, y_{0}\right)
$$

(iv) Let $\lambda_{1}$ be the smallest non-null eigenvalue of the infinitesimal generator $G$. Let $\alpha=u_{1}$ be the corresponding eigenvector, that is, satisfying, $-G u_{1}=\lambda_{1} u_{1}$ with boundary conditions $u_{1}(0)=u_{1}(1)=0$. Then, $c=\lambda u_{1}$. The new KB operator associated to the 
transformed process $\left(\bar{x}_{t} ; t \geq 0\right)$ is

$$
\bar{G}(\cdot)=\frac{1}{\alpha} G(\alpha) \cdot+\tilde{G}(\cdot)=-\lambda_{1} \cdot+\tilde{G}(\cdot),
$$

obtained while killing the sample paths of the process $\left(\tilde{x}_{t} ; t \geq 0\right)$ governed by $\widetilde{G}$ at constant death rate $d=\lambda_{1}$. The transition probability of the transformed stochastic process $\left(\bar{x}_{t} ; t \geq 0\right)$ is

$$
\bar{p}(x ; t, y)=\frac{u_{1}(y)}{u_{1}(x)} p(x ; t, y) .
$$

Define $\tilde{p}(x ; t, y)=e^{\lambda_{1} t} \bar{p}(x ; t, y)$. It is the transition probability of the process $\left(\tilde{x}_{t} ; t \geq 0\right)$ governed by $\widetilde{G}$; it corresponds to the original process $\left(x_{t} ; t \geq 0\right)$ conditioned on never hitting the boundaries $\{0,1\}$ (the so-called $Q$-process of $\left(x_{t} ; t \geq 0\right)$, see [19]). It is simply obtained from $\left(x_{t} ; t \geq 0\right)$ by adding the additional drift term $\left(u_{1}^{\prime} / u_{1}\right) g^{2}$ to $f$, where $u_{1}$ is the eigenvector of $G$ associated to its smallest non-null eigenvalue. The determination of $\alpha=u_{1}$ is a SturmLiouville problem. When $t$ is large, to the dominant order

$$
p(x ; t, y) \sim e^{-\lambda_{1} t} \frac{u_{1}(x) v_{1}(y)}{\left\langle u_{1}, v_{1}\right\rangle}
$$

where $v_{1}$ is the Yaglom limit law of $\left(x_{t} ; t \geq 0\right)$. Therefore

$$
\tilde{p}(x ; t, y) \sim e^{\lambda_{1} t} \frac{u_{1}(y)}{u_{1}(x)} e^{-\lambda_{1} t} \frac{u_{1}(x) v_{1}(y)}{\left\langle u_{1}, v_{1}\right\rangle}=\frac{u_{1}(y) v_{1}(y)}{\left\langle u_{1}, v_{1}\right\rangle}
$$

Thus, the limit law of the $Q$-process $\left(\tilde{x}_{t} ; t \geq 0\right)$ is the normalized Hadamard product of the eigenvectors $u_{1}$ and $v_{1}$ associated, respectively, to $G$ and $G^{*}$. On the other hand, the limit law of $\left(\tilde{x}_{t} ; t \geq 0\right)$ is directly given by

$$
\tilde{p}(x ; t, y) \underset{t \rightarrow \infty}{\longrightarrow} \tilde{p}(y)=\frac{1}{Z g^{2}(y)} e^{2 \int_{0}^{y}\left(\left(f(z)+\left(\left(u_{1}^{\prime} / u_{1}\right) g^{2}\right)(z)\right) /\left(g^{2}(z)\right)\right) d z}=\frac{u_{1}^{2}(y)}{Z g^{2}(y)} e^{2 \int_{0}^{y}\left(f(z) /\left(g^{2}(z)\right)\right) d z,}
$$

where $\mathrm{Z}$ is the appropriate normalizing constant. Comparing (2.77) and (2.78)

$$
v_{1}(y)=\frac{u_{1}(y)}{g^{2}(y)} \mathrm{e}^{2 \int_{0}^{y}\left(f(z) /\left(g^{2}(z)\right)\right) d z}=u_{1}(y) m(y)
$$

The eigenvector $v_{1}$ associated to $G^{*}$ is, therefore, equal to the eigenvector $u_{1}$ associated to $G$ times the speed density of $\left(x_{t} ; t \geq 0\right)$. 
When dealing for example with the neutral Wright-Fisher diffusion, it is known that $\lambda_{1}=1$ with $u_{1}=x(1-x)$ and $v_{1} \equiv 1$ (see Section 4.3, example (ii)). The $Q$-process $\left(\tilde{x}_{t} ; t \geq 0\right)$ in this case obeys

$$
d \tilde{x}_{t}=\left(1-2 \tilde{x}_{t}\right) d \bar{t}+\sqrt{\tilde{x}_{t}\left(1-\tilde{x}_{t}\right)} d w_{t}
$$

with the stabilizing drift toward 1/2: $\tilde{f}(x)=\left(u_{1}^{\prime} / u_{1}\right) g^{2}(x)=1-2 x$.

The limit law of the $Q$-process $\left(\tilde{x}_{t} ; t \geq 0\right)$ in this case is $6 y(1-y)$. The latter conditioning is more stringent than the Yaglom conditioning and so the limiting law has more mass away from the boundaries (compare with the uniform Yaglom limit). For additional similar examples in the context of WF diffusions and related ones, see [20].

\subsection{Branching and the Reciprocal Doob Transform}

Clearly, starting from the killed diffusion process with infinitesimal generator $\bar{G}(\cdot)=-d(x)$. $+\widetilde{G}(\cdot)$ and applying the reciprocal Doob transform defined by

$$
\tilde{\alpha}(x)=\frac{1}{\alpha(x)}
$$

leads to $\tilde{\alpha}(x)^{-1} \bar{G}(\widetilde{\alpha}(x) \cdot)=G(\cdot)$. Indeed,

$$
\begin{aligned}
\tilde{\alpha}(x)^{-1} \bar{G}(\widetilde{\alpha}(x) \cdot) & =-d(x) \cdot+\widetilde{\alpha}(x)^{-1} \widetilde{G}(\widetilde{\alpha}(x) \cdot), \\
\widetilde{\alpha}(x)^{-1} \tilde{G}(\widetilde{\alpha}(x) \cdot) & =\widetilde{\alpha}(x)^{-1} \tilde{G}(\widetilde{\alpha}(x)) \cdot+\widetilde{G}(\cdot)+\frac{1}{\widetilde{\alpha}} g^{2} \widetilde{\alpha}^{\prime} \partial_{x} \\
& =d(x) \cdot+G(\cdot),
\end{aligned}
$$

because

$$
\begin{gathered}
\tilde{\alpha}(x)^{-1} \tilde{G}(\tilde{\alpha}(x))=+d(x), \\
\frac{1}{\tilde{\alpha}} g^{2} \widetilde{\alpha}^{\prime} \partial_{x}=\alpha g^{2}\left(\frac{1}{\alpha}\right)^{\prime} \partial_{x}=-\frac{\alpha^{\prime}}{\alpha} g^{2} \partial_{x} .
\end{gathered}
$$

Note that $\bar{G}(\tilde{\alpha}(x))=\tilde{G}(\tilde{\alpha}(x))-d(x) \tilde{\alpha}(x)=0$.

This suggests that starting from a diffusion process with infinitesimal generator $\tilde{G}$ (without its killing part) and applying the reciprocal Doob transform $\tilde{\alpha}(x)=1 /(\alpha(x)$ ), one ends up with a modified process whose infinitesimal generator is

$$
\overline{\widetilde{G}}(\cdot)=G(\cdot)+b(x),
$$

where $G(\cdot)=\widetilde{G}(\cdot)-\left(\alpha^{\prime} / \alpha\right) g^{2}(x) \partial_{x}$ and

$$
b(x)=\widetilde{\alpha}(x)^{-1} \tilde{G}(\widetilde{\alpha}(x))=d(x)>0
$$


is now a pure birth rate. Note that $\tilde{\alpha}$ is now a subharmonic function for $\tilde{G}$ because $-\tilde{G}(\tilde{\alpha})=$ $-b \tilde{\alpha} \leq 0$.

Let $\tilde{\beta}(x):=\tilde{\alpha}(x)^{-1}=\alpha(x)$. Because $G(\alpha(x))=-\alpha(x) b(x)$, we have

$$
\overline{\widetilde{G}}(\tilde{\beta}(x))=G(\tilde{\beta}(x))+b(x) \tilde{\beta}(x)=0,
$$

and so $\tilde{\beta}(x)>0$ is harmonic for $\overline{\widetilde{G}}$ : Doob-transforming $\overline{\widetilde{G}}$ using $\tilde{\beta}$, we get

$$
\tilde{\beta}^{-1} \overline{\widetilde{G}}(\widetilde{\beta} \cdot)=b(x) \cdot+\tilde{\beta}^{-1} G(\widetilde{\beta} \cdot)=\widetilde{G}(\cdot),
$$

which is the infinitesimal generator of the original diffusion process.

Under our assumptions, both process $x_{t}$ and $\tilde{x}_{t}$ with infinitesimal generators $G$ and $\tilde{G}$ are nonconservative diffusion processes with absorbing barriers. Further, $b(x)>0$ is bounded above. Therefore, $b(x)$ may be written as

$$
b(x)=b_{*}(\mu(x)-1)
$$

where $b_{*}=\max _{x} b(x)>0$ and $\mu(x) \in[1,2]$.

The process with infinitesimal generator $\overline{\widetilde{G}}$ is now a pure binary branching diffusion process. For this class of models, an initial particle started at $x$ obeys a diffusion process with infinitesimal generator $G$, absorbed when it hits the boundaries. At some random (mean $b_{*}$ ) exponential time, this particle dies, giving birth in the process to a random number $M(x)$ (either 1 or 2) of daughter particles started where the mother particle died and diffusing independently as their mother did and so forth for the subsequent generation particles. We have $\operatorname{E} M(x)=\mu(x)$.

The process with infinitesimal generator $\overline{\widetilde{G}}$ is, thus, a branching diffusion with supercritical binary splitting mechanism $(\mu(x)>1)$. There is, therefore, a competition between the branching phenomenon that leads to an exponential increase of the number of particles in the system and the absorption at the boundaries of the living particles.

Let $N_{t}(x)$ be the global number of particles which are alive in the system at each time $t$, descending from an Eve particle started at $x$, and let

$$
T(x)=\inf \left(t>0: N_{t}(x)=0\right)
$$

be the global extinction time of the population. Under our assumptions, this branching model fits to the general formalism for branching diffusion developed in $([9,10])$ from which we conclude

$$
\mathbf{P}\left(T(x)<\infty, N_{t}(x)=0 \quad \forall t \geq T(x)\right)=1,
$$

uniformly in $x$. This means the global extinction of the particle system under concern: In the tradeoff between branching and absorption at the boundaries, the system gets eventually extinct with probability 1 in finite time. We shall develop a typical example arising in population genetics in the subsequent sections. 


\section{The Wright-Fisher Example}

In this section, we briefly and informally recall that the celebrated WF diffusion process with or without a drift may be viewed as a scaling limit of a simple two alleles discrete spacetime branching process preserving the total number $N$ of individuals in the subsequent generations (see $[8,12,21]$ for example).

\subsection{The Neutral Wright-Fisher Model}

Consider a discrete-time Galton Watson branching process preserving the total number of individuals in each generation. We start with $N$ individuals. The initial reproduction law is defined as follows: let $\left|\mathbf{k}_{N}\right|:=\sum_{m=1}^{N} k_{m}=N$ and $\mathbf{k}_{N}:=\left(k_{1}, \ldots, k_{N}\right)$ be integers. Assume that the first-generation random offspring numbers $\boldsymbol{v}_{N}:=\left(v_{N}(1), \ldots, v_{N}(N)\right)$ admit the following joint exchangeable polynomial distribution on the discrete simplex $\left|\mathbf{k}_{N}\right|=N$ :

$$
\mathbf{P}\left(v_{N}=\mathbf{k}_{N}\right)=\frac{N ! \cdot N^{-N}}{\prod_{n=1}^{N} k_{n} !} .
$$

This distribution can be obtained by conditioning $N$ independent Poisson distributed random variables on summing to $N$. Assume subsequent iterations of this reproduction law are independent so that the population is with constant size for all generations.

Let $N_{r}(n)$ be the offspring number of the $n$ first individuals at the discrete generation $r \in \mathbf{N}_{0}$ corresponding to (say) allele $A_{1}$ (the remaining number $N-N_{r}(n)$ counts the number of alleles $A_{2}$ at generation $r$ ). This sibship process is a discrete-time Markov chain with binomial transition probability given by

$$
\mathbf{P}\left(N_{r+1}(n)=k^{\prime} \mid N_{r}(n)=k\right)=\left(\begin{array}{l}
N \\
k^{\prime}
\end{array}\right)\left(\frac{k}{N}\right)^{k^{\prime}}\left(1-\frac{k}{N}\right)^{N-k^{\prime}} .
$$

Assume next that $n=[N x]$, where $x \in(0,1)$. Then, as well known, the dynamics of the continuous space-time rescaled process $x_{t}:=N_{[N t]}(n) / N, t \in \mathbf{R}_{+}$can be approximated for large $N$, to the leading term in $N^{-1}$, by a Wright-Fisher-Itô diffusion on $[0,1]$ (the purely random genetic drift case)

$$
d x_{t}=\sqrt{x_{t}\left(1-x_{t}\right)} d w_{t}, \quad x_{0}=x
$$

Here, $\left(w_{t} ; t \geq 0\right)$ is a standard Wiener process. For this scaling limit process, a unit laps of time $t=1$ corresponds to a laps of time $N$ for the original discrete-time process, thus time is measured in units of $N$. If the initial condition is $x=N^{-1}, x_{t}$ is the diffusion approximation of the offspring frequency of a singleton at generation $[N t]$.

Equation (3.3) is a one-dimensional diffusion as in $(2.1)$ on $[0,1]$, with zero drift $f(x)=0$ and volatility $g(x)=\sqrt{x(1-x)}$. This diffusion is already in natural coordinate, and so $\varphi(x)=x$. The scale function is $x$ and the speed measure $[x(1-x)]^{-1} d x$. One can check that both boundaries are exit in this case: the stopping time is $\tau_{x}=\tau_{x, 0} \wedge \tau_{x, 1}$ where $\tau_{x, 0}$ is the extinction time and $\tau_{x, 1}$ the fixation time. The corresponding infinitesimal generators are $G(\cdot)=(1 / 2) x(1-x) \partial_{x}^{2}(\cdot)$ and $G^{*}(\cdot)=(1 / 2) \partial_{y}^{2}(y(1-y) \cdot)$. 


\subsection{Nonneutral Cases}

Two alleles Wright-Fisher models (with non-null drifts) can be obtained by considering the binomial transition probabilities $\operatorname{bin}\left(N, p_{N}\right)$

$$
\mathbf{P}\left(N_{r+1}(n)=k^{\prime} \mid N_{r}(n)=k\right)=\left(\begin{array}{l}
N \\
k^{\prime}
\end{array}\right)\left(p_{N}\left(\frac{k}{N}\right)\right)^{k^{\prime}}\left(1-p_{N}\left(\frac{k}{N}\right)\right)^{N-k^{\prime}},
$$

where

$$
p_{N}(x): x \in(0,1) \longrightarrow(0,1)
$$

is now some state-dependent probability (which is different from the identity $x$ ) reflecting some deterministic evolutionary drift from the allele $A_{1}$ to the allele $A_{2}$. For each $r$, we have

$$
\begin{gathered}
\mathbf{E}\left(N_{r+1}(n) \mid N_{r}(n)=k\right)=N p_{N}\left(\frac{k}{N}\right), \\
\sigma^{2}\left(N_{r+1}(n) \mid N_{r}(n)=k\right)=N p_{N}\left(\frac{k}{N}\right)\left(1-p_{N}\left(\frac{k}{N}\right)\right),
\end{gathered}
$$

which is amenable to a diffusion approximation in terms of $x_{t}:=N_{[N t]}(n) / N, t \in \mathbf{R}_{+}$under suitable conditions.

For instance, taking $p_{N}(x)=\left(1-\pi_{2, N}\right) x+\pi_{1, N}(1-x)$, where $\left(\pi_{1, N}, \pi_{2, N}\right)$ are small ( $N$-dependent) mutation probabilities from $A_{1}$ to $A_{2}$ ( $A_{2}$ to $A_{1}$, resp.). Assuming that $\left(N \cdot \pi_{1, N}, N \cdot \pi_{2, N}\right) \underset{N \rightarrow \infty}{\rightarrow}\left(u_{1}, u_{2}\right)$, leads after scaling to the drift of WF model with positive mutations rates $\left(u_{1}, u_{2}\right)$.

Taking

$$
p_{N}(x)=\frac{\left(1+s_{1, N}\right) x}{\left(1+s_{1, N} x+(1-x)\left(1+s_{2, N}\right)\right)}
$$

where $s_{i, N}>0$ are small $N$-dependent selection parameter satisfying $N \cdot s_{i, N} \underset{N \rightarrow \infty}{\rightarrow} \sigma_{i}>0, i=$ 1,2 , leads, after scaling, to the WF model with selective drift $\sigma x(1-x)$, where $\sigma:=\sigma_{1}-\sigma_{2}$. Essentially, the drift $f(x)$ is a large $N$ approximation of the bias: $N\left(p_{N}(x)-x\right)$. The WF diffusion with selection is thus

$$
d x_{t}=\sigma x_{t}\left(1-x_{t}\right) d t+\sqrt{x_{t}\left(1-x_{t}\right)} d w_{t}
$$

where time is measured in units of $N$. Letting $\theta_{t}=N t$ define a new time scale with inverse $t_{\theta}=\theta / N$, the time-changed process $y_{\theta}=x_{\theta / N}$ now obeys the SDE

$$
d y_{\theta}=s y_{\theta}\left(1-y_{\theta}\right) d \theta+\sqrt{\frac{1}{N} y_{\theta}\left(1-y_{\theta}\right)} d w_{\theta}
$$

with a small diffusion term. Here, $s=s_{1}-s_{2}$ and time $\theta$ is the usual time clock. 
The WF diffusion with selection (3.8) tends to drift to $\circ=1\left(\circ=0\right.$, resp.) if allele $A_{1}$ is selectively advantageous over $A_{2}: \sigma_{1}>\sigma_{2}\left(\sigma_{1}<\sigma_{2}\right.$, resp.) in the following sense: if $\sigma>0$ $(<0$, resp. $)$, the fixation probability at $\circ=1$, which is [15]

$$
\mathbf{P}\left(\tau_{x, 1}<\tau_{x, 0}\right)=\frac{1-e^{-2 \sigma x}}{1-e^{-2 \sigma}}
$$

increases (decreases) with $\sigma$ taking larger (smaller) values. Putting $x=1 / N$, the fixation probability at 1 of an allele $A_{1}$ mutant is of order: $2 \sigma / N$; see [15].

\section{The Neutral WF Model}

In this section, we particularize the general ideas developed in the introductory Section 2 to the neutral WF diffusion (3.3) and draw some straightforward conclusions most of which are known which illustrate the use of Doob transforms.

\subsection{Explicit Solutions of the Neutral KBE and KFE}

As shown by Kimura in [22], it turns out that both Kolmogorov equations are exactly solvable, in this case, using spectral theory. Indeed, the solutions involve a series expansion in terms of eigenfunctions of the KB and KF infinitesimal generators with discrete eigenvalues spectrum. We now consider the specific neutral WF model.

With $z \in(-1,1)$, let $\left(P_{k}(z) ; k \geq 0\right)$ be the degree- $(k+1)$ Gegenbauer polynomials solving $\left(1-z^{2}\right) P_{k}^{\prime \prime}(z)+k(k+1) P_{k}(z)=0$ with $P_{k}^{\prime}( \pm 1)=\mp(1 / 2), \quad k \geq 1$; we let $P_{0}(z):=$ $(1-z) / 2$. When $k \geq 1$, we have $P_{k}( \pm 1)=0$ and so $P_{k}(z)=\left(1-z^{2}\right) Q_{k}(z)$, where $Q_{k}(z)$ is a polynomial with degree $k-1$ satisfying $Q_{k}(-1)=(-1)^{k-1}$ and $Q_{k}(1)=1$. With $x \in(0,1)$, let $\left(u_{k}(x) ; k \geq 0\right)$ be defined by: $u_{k}(x)=P_{k}(1-2 x)$. These polynomials clearly constitute a system of eigenfunctions for the KB operator $-G=-(1 / 2) x(1-x) \partial_{x}^{2}$ with eigenvalues $\lambda_{k}=$ $(k(k+1)) / 2, \quad k \geq 0$, thus with $-G\left(u_{k}(x)\right)=\lambda_{k} u_{k}(x)$. In particular, $u_{0}(x)=x, u_{1}(x)=x-x^{2}$, $u_{2}(x)=x-3 x^{2}+2 x^{3}, u_{3}(x)=x-6 x^{2}+10 x^{3}-5 x^{4}, u_{4}(x)=x-10 x^{2}+30 x^{3}-35 x^{4}+14 x^{5}, \ldots$ With $k \geq 1$, we have $u_{k}(0)=u_{k}(1)=0$ and $u_{k}^{\prime}(0)=1$ and $u_{k}^{\prime}(1)=-1$.

The eigenfunctions of the KF operator $G^{*}(\cdot)=(1 / 2) \partial_{x}^{2}[y(1-y) \cdot]$ are given by $v_{k}(y)=$ $m(y) \cdot u_{k}(y), k \geq 0$, where the Radon measure of weights $m(y) d y$ is the speed measure: $m(y) d y=d y /(y(1-y))$, for the same eigenvalues. For instance, $v_{0}(y)=1 /(1-y), v_{1}(y)=1$, $v_{2}(y)=1-2 y, v_{3}(y)=1-5 y+5 y^{2}, v_{4}(y)=1-9 y+21 y^{2}-14 y^{3}, \ldots$

Although $\lambda_{0}=0$ really constitutes an eigenvalue, only $v_{0}(y)$ is not a polynomial. When $k \geq 1$, from their definition, the $u_{k}(x)$ polynomials satisfy $u_{k}(0)=u_{k}(1)=0$ in such a way that $v_{k}(y)=m(y) \cdot u_{k}(y), k \geq 1$ is a polynomial with degree $k-1$.

Let $\langle f, g\rangle_{m}=\int_{0}^{1} f(x) g(x) m(x) d x$. We note that $\left\langle v_{j}, u_{k}\right\rangle=\left\langle u_{j}, u_{k}\right\rangle_{m}=0$ if $j \neq k$ and the system $u_{k}(x) ; k \geq 1$ is a complete orthogonal set of eigenvectors. Therefore, for any square-integrable function $\psi(x) \in L_{2}([0,1], m(y) d y)$ admitting a decomposition in the basis $u_{k}(x), k \geq 1$.

$$
\mathrm{E} \psi\left(x_{t}\right)=\sum_{k \geq 1} c_{k} e^{-\lambda_{k} t} u_{k}(x), \quad \text { where } c_{k}=\frac{\left\langle\psi, u_{k}\right\rangle_{m}}{\left\langle v_{k}, u_{k}\right\rangle}
$$

where $\psi(x)=\sum_{k \geq 1} c_{k} u_{k}(x)$. This series expansion solves the KBE: $\partial_{t} u=G(u) ; u(x, 0)=\psi(x)$ where $u=u(x, t):=\mathrm{E} \psi\left(x_{t}\right)$. 
Moreover, the transition probability density $p(x ; t, y)$ of the neutral WF models admits the spectral expansion

$$
p(x ; t, y)=\sum_{k \geq 1} b_{k} e^{-\lambda_{k} t} u_{k}(x) v_{k}(y), \quad \text { where } b_{k}=\frac{1}{\left\langle v_{k}, u_{k}\right\rangle} .
$$

Starting from $x$, the cumulated probability masses by time $t$ at the exit boundaries $\{0,1\}$ are, respectively, (see [17])

$$
\begin{aligned}
& \frac{1}{2} \int_{0}^{t} p(x ; s, 0) d s=\sum_{k \geq 1} \frac{b_{k}}{2 \lambda_{k}}\left(1-e^{-\lambda_{k} t}\right) u_{k}(x) v_{k}(0), \\
& \frac{1}{2} \int_{0}^{t} p(x ; s, 1) d s=\sum_{k \geq 1} \frac{b_{k}}{2 \lambda_{k}}\left(1-e^{-\lambda_{k} t}\right) u_{k}(x) v_{k}(1),
\end{aligned}
$$

which tend as $t \rightarrow \infty$ toward the extinction and fixation probabilities, namely, here $\mathbf{P}\left(\tau_{x, 0}<\right.$ $\infty)=\mathbf{P}\left(\tau_{x, 0}<\tau_{x, 1}\right)=1-x$ and $\mathbf{P}\left(\tau_{x, 1}<\infty\right)=x$. Because $v_{k}(0)=1$ and $v_{k}(1)=(-1)^{k-1}$, we get the identities

$$
\begin{gathered}
1-x=\sum_{k \geq 1} \frac{b_{k}}{2 \lambda_{k}} u_{k}(x) \quad \text { or } \frac{1}{x}=\sum_{k \geq 1} \frac{b_{k}}{2 \lambda_{k}} v_{k}(x), \\
x=\sum_{k \geq 1} \frac{(-1)^{k-1} b_{k}}{2 \lambda_{k}} u_{k}(x) \quad \text { or } \quad \frac{1}{1-x}=\sum_{k \geq 1} \frac{(-1)^{k-1} b_{k}}{2 \lambda_{k}} v_{k}(x),
\end{gathered}
$$

leading to the relationship $\sum_{k \geq 1}\left((-1)^{k-1} b_{k} / 2 \lambda_{k}\right)=1$.

The series expansion for $p(x ; t, y)$ solves the KFE of the WF model. The transition density $p(x ; t, y)$ is reversible with respect to the speed density since for $0<x, y<1$

$$
m(x) p(x ; t, y)=m(y) p(y ; t, x)=\sum_{k \geq 1} b_{k} e^{-\lambda_{k} t} v_{k}(x) v_{k}(y) .
$$

The measures $v_{k}(y) d y, k \geq 1$ are not probability measures because the $v_{k}(y)$ are not necessarily positive over $[0,1]$. This decomposition is not a mixture. We have $\left\langle v_{k}, u_{k}\right\rangle=$ $\left\|u_{k}\right\|_{2, m}^{2}$ the 2-norm for the weight function $m$. We notice that $\left\langle v_{0}, u_{0}\right\rangle=\int_{0}^{1}(y /(1-y)) d y=\infty$ so that $c_{0}=b_{0}=0$ although $\lambda_{0}=0$ is indeed an eigenvalue, the above sums should be started at $k=1$ (expressing the lack of an invariant measure for the WF model as a result of its absorption at the boundaries).

We have $\mathbf{P}\left(\tau_{x}>t\right)=\int_{0}^{1} \mathbf{P}\left(x_{t} \in d y\right)$ and so

$$
\rho_{t}(x):=\mathbf{P}\left(\tau_{x}>t\right)=\sum_{k \geq 1} \frac{\int_{0}^{1} v_{k}(y) d y}{\left\langle v_{k}, u_{k}\right\rangle} e^{-\lambda_{k} t} u_{k}(x)
$$

is the exact tail distribution of the absorption time. 
International Journal of Stochastic Analysis

Since $v_{1}(y)=1$, to the leading order in $t$, for large time

$$
\mathbf{P}\left(x_{t} \in d y\right)=6 e^{-t} \cdot x(1-x) d y+\mathcal{O}\left(e^{-3 t}\right)
$$

which is independent of $y$. Integrating over $y, \rho_{t}(x):=\mathbf{P}\left(\tau_{x}>t\right) \sim 6 e^{-t} \cdot x(1-x)$ so that the conditional probability

$$
\mathbf{P}\left(x_{t} \in d y \mid \tau_{x}>t\right) \underset{t \rightarrow \infty}{\sim} d y
$$

is asymptotically uniform in the Yaglom limit. As time passes by, given absorption did not occur in the past, $x_{t} \stackrel{d}{\rightarrow} x_{\infty}$ (as $\left.t \rightarrow \infty\right)$ which is a uniformly distributed random variable on $[0,1]$.

\subsection{Additive Functionals for the Neutral WF}

Let $\left(x_{t} ; t \geq 0\right)$ be the WF diffusion model defined by (3.3) on the interval $I=[0,1]$, where both endpoints are absorbing (exit). We wish to evaluate the additive quantities

$$
\alpha(x)=\mathbf{E}\left(\int_{0}^{\tau_{x}} c\left(x_{s}\right) d s+d\left(x_{\tau_{x}}\right)\right)
$$

where functions $c$ and $d$ are both nonnegative. With $G=(1 / 2) x(1-x) \partial_{x}^{2}, \alpha(x)$ solves

$$
\begin{gathered}
-G(\alpha)=c \quad \text { if } x \in I, \\
\alpha=d \quad \text { if } x \in \partial I .
\end{gathered}
$$

Take $c=\lim _{\varepsilon \downarrow 0}(1 / 2 \varepsilon) \mathbf{1}(x \in(y-\varepsilon, y+\varepsilon))=: \delta_{y}(x)$ and $d=0$, when $y \in I$ : in this case, $\alpha:=\mathfrak{g}(x, y)$ is the Green function. The solution takes the simple form

$$
\begin{gathered}
\mathfrak{g}(x, y)=2 \frac{x}{y} \quad \text { if } x<y \\
\mathfrak{g}(x, y)=2 \frac{1-x}{1-y} \quad \text { if } x>y
\end{gathered}
$$

The Green function solves the above general problem of evaluating additive functionals $\alpha(x)$

$$
\begin{gathered}
\alpha(x)=\int_{I} g(x, y) c(y) d y \quad \text { if } x \in I, \\
\alpha=d \quad \text { if } x \in \partial I .
\end{gathered}
$$


As a Few Examples

(1) Let $c=1$ and $d=0$ : here, $\alpha(x)=\mathbf{E}\left(\tau_{x}\right)$ is the mean time of absorption (average time spent in $I$ before absorption). The solution is (the Crow and Kimura formula, see [2])

$$
\alpha(x)=2 x \int_{x}^{1} \frac{d y}{y}+2(1-x) \int_{0}^{x} \frac{d y}{1-y}=-2(x \log x+(1-x) \log (1-x)) .
$$

(2) Let $c=0$ and $d(0)=\mathbf{1}(0=1)$. Let $\alpha(x)=\mathbf{P}\left(x_{t}\right.$ first hits $[0,1]$ at $\left.1 \mid x_{0}=x\right)$. Then, $\alpha(x)$ is a $G$-harmonic function solution to $G(\alpha)=0$, with boundary conditions $\alpha(0)=0$ and $\alpha(1)=1$. The solution for WF model is: $\alpha(x)=x$. Stated differently, $x=\mathbf{P}\left(\tau_{x, 1}<\tau_{x, 0}\right)$ is the probability that the exit time at $\mathrm{o}=1$ is less than the one at $\mathrm{o}=0$, starting from $x$.

On the contrary, choosing $\alpha(x)$ to be a G-harmonic function with boundary conditions $\alpha(0)=$ 1 and $\alpha(1)=0, \alpha(x)=\mathbf{P}\left(x_{t}\right.$ first hits $[0,1]$ at $\left.0 \mid x_{0}=x\right)=1-x$. Thus, $1-x=\mathbf{P}\left(\tau_{x, 0}<\tau_{x, 1}\right)$.

(3) Let $c\left(x_{s}\right)=2 x_{s}\left(1-x_{s}\right)$ measure the heterozygosity of the WF process at time $s$ and assume $d(0)=d(1)=1$. A remarkable thing is that the average heterozygosity over the sample paths is

$$
\alpha(x)=\mathbf{E}\left(\int_{0}^{\tau_{x}} c\left(x_{s}\right) d s\right)=4 x \int_{x}^{1}(1-y) d y+4(1-x) \int_{0}^{x} y d y=2 x(1-x),
$$

which is the initial heterozygosity of the population.

\subsection{Transformation of WF Sample Paths, [3]}

With $p(x ; t, y)$ the transition probability density of WF model, define a new $\alpha$-transformed stochastic process $\left(\bar{x}_{t} ; t \geq 0\right)$ by its transition probability

$$
\bar{p}(x ; t, y)=\frac{\alpha(y)}{\alpha(x)} p(x ; t, y) \text {. }
$$

(i) Conditioning WF on exit at some boundary. Assume first $\alpha$ solves $-G(\alpha)=0$ with boundary conditions $\alpha(0)=0$ and $\alpha(1)=1$; hence, $\alpha$ reads $\alpha(x)=x$. In this case, $\tilde{\tau}_{x, \partial}=\infty$ (no killing), and so $\bar{\tau}_{x}:=\widetilde{\tau}_{x}$ is the absorption time for a process $\left(\tilde{x}_{t} ; t \geq 0\right)$ governed by a new SDE with a drift term. The new process $\left(\tilde{x}_{t} ; t \geq 0\right)$ is just $\left(x_{t} ; t \geq 0\right)$ conditioned on exiting at $\circ=1$. The boundary 1 is exit whereas 0 is entrance. Thus, the model for $\left(\tilde{x}_{t} ; t \geq 0\right)$ becomes $d \tilde{x}_{t}=\left(1-\tilde{x}_{t}\right) d t+\sqrt{\tilde{x}_{t}\left(1-\tilde{x}_{t}\right)} d w_{t}, \tilde{x}_{0}=x \in(0,1)$ now with linear drift $\tilde{f}(x)=1-x$ and $g(x)=\sqrt{x(1-x)}$. Its transition probability is

$$
\bar{p}_{1}(x ; t, y)=\frac{y}{x} p(x ; t, y),
$$

where the subscript 1 indicates that this is the conditional transition probability of sample paths whose exit is necessarily at the boundary 1 .

Assuming now $\alpha$ solves $-G(\alpha)=0$ if $x \in I$ with boundary conditions $\alpha(0)=1$ and $\alpha(1)=0$, the new process $\left(\tilde{x}_{t} ; t \geq 0\right)$ is just $\left(x_{t} ; t \geq 0\right)$ conditioned on exiting at $x=0$. Boundary 0 is exit, whereas 1 is entrance; in this case, $\alpha$ is $\alpha(x)=1-x$. Thus, the model for $\left(\tilde{x}_{t} ; t \geq 0\right)$ 
becomes $d \tilde{x}_{t}=-\tilde{x}_{t} d t+\sqrt{\tilde{x}_{t}\left(1-\tilde{x}_{t}\right)} d w_{t}, \tilde{x}_{0}=x \in(0,1)$ with $\tilde{f}(x)=-x$ and $g(x)=\sqrt{x(1-x)}$. Its transition probability is

$$
\bar{p}_{0}(x ; t, y)=\frac{1-y}{1-x} p(x ; t, y),
$$

where the subscript 0 indicates that this is the conditional transition probability of WF sample paths whose exit now is at $\mathrm{o}=0$. Recalling that, starting from $x,\left(x_{t} ; t \geq 0\right)$ gets absorbed at $\circ=1(0$, resp.) with probability $x(1-x$, resp. $)$, we recover that

$$
p(x ; t, y)=x \cdot \bar{p}_{1}(x ; t, y)+(1-x) \cdot \bar{p}_{0}(x ; t, y) .
$$

Using the solution to KFE for $p$, we obtain an expression for both $\bar{p}_{1}(x ; t, y)$ and $\bar{p}_{0}(x ; t, y)$, simply by premultiplying it by the corresponding right factor. Integrating the results over $y$, we get the conditional tail distributions of the exit times at $\circ=1$ or 0 , given the exit is at $\circ=1$ or 0 .

Exploiting the large time behavior of $p(x ; t, y)$, to the first order in $t$, we get

$$
\begin{aligned}
& \bar{p}_{1}(x ; t, y) \sim 6 e^{-t} \cdot(1-x) y, \\
& \bar{p}_{0}(x ; t, y)=6 e^{-t} \cdot x(1-y) .
\end{aligned}
$$

Integrating over $y, \bar{\rho}_{t, 1}(x):=\mathbf{P}_{1}\left(\widetilde{\tau}_{x}>t\right) \sim 3 e^{-t} \cdot(1-x)$ and $\bar{\rho}_{t, 0}(x):=\mathbf{P}_{0}\left(\widetilde{\tau}_{x}>t\right) \sim 3 e^{-t} \cdot x$ are the large time behaviors of the absorption times at 1 and 0 , respectively. Using this, we get the large time behaviors of the conditional probabilities

$$
\begin{gathered}
\mathbf{P}_{1}\left(\tilde{x}_{t} \in d y \mid \tilde{\tau}_{x}>t\right) \sim 2 y d y, \\
\mathbf{P}_{0}\left(\tilde{x}_{t} \in d y \mid \tilde{\tau}_{x}>t\right) \sim 2(1-y) d y,
\end{gathered}
$$

where we recognize the densities of specific beta-distributed random variables. Specifically, we conclude that as time passes by, given absorption occurs at $0=1$ and given it has not occurred in the past, $\tilde{x}_{t} \stackrel{d}{\rightarrow}$ beta $(2,1)$ distribution on $[0,1]$. Similarly, given absorption occurs at $\circ=0$ and given it has not occurred previously, $\tilde{x}_{t} \stackrel{d}{\rightarrow}$ beta $(1,2)$ distribution on $[0,1]$.

In the previously displayed formula, $\widetilde{\tau}_{x}$ is just the exit time at $0=1$ (at $\circ=$ 0 , resp.) of the conditional transformed WF diffusions. Let $\widetilde{\alpha}(x):=\mathbf{E}\left(\widetilde{\tau}_{x}\right)$. Then, with $\bar{G}(\cdot)=$ $(1 / \alpha(x)) G(\alpha(x) \cdot), \tilde{\alpha}(x)$ solves $-\bar{G}(\widetilde{\alpha})=1$, whose explicit solution is

$$
\tilde{\alpha}(x)=\frac{1}{\alpha(x)} \int_{0}^{1} \mathfrak{g}(x, y) \alpha(y) d y,
$$

in terms of $\mathfrak{g}(x, y)$, the Green function of $\left(x_{t} ; t \geq 0\right)$. For the WF model conditioned on exit at $\circ=1(0$, resp.), we find, respectively, the Kimura and Ohta's formulae in [23]

$$
\begin{gathered}
\tilde{\alpha}_{1}(x)=-\frac{2}{x}(1-x) \log (1-x), \\
\tilde{\alpha}_{0}(x)=-\frac{2}{1-x} x \log x .
\end{gathered}
$$


This result could have been guessed by observing that $x \widetilde{\alpha}_{1}(x)+(1-x) \widetilde{\alpha}_{0}(x)$ is the expected absorption time of the original WF model. When $x \rightarrow 0^{+}$, (resp., $\left.x \rightarrow 1^{-}\right)$, it takes an average time 2 to reach $1(0$, resp.) for the WF model conditioned on exit at $\circ=1(0$, resp.).

(ii) Selection of WF sample paths with large heterozygosity. Assume that $\alpha$ now solves $-G(\alpha)=2 x(1-x)$ if $x \in I$ with boundary conditions $\alpha(0)=\alpha(1)=0$. Then, $\alpha=2 x(1-x)$ and this $\alpha$ is the right eigenvector of $-G$ associated to the smallest positive eigenvalue $\lambda_{1}=1$ of the neutral WF model. In this case study, one selects sample paths of $\left(x_{t} ; t \geq 0\right)$ with large heterozygosity. The dynamics of $\left(\tilde{x}_{t} ; t \geq 0\right)$ in (2.38) is

$$
d \tilde{x}_{t}=\left(1-2 \tilde{x}_{t}\right) d t+\sqrt{\tilde{x}_{t}\left(1-\tilde{x}_{t}\right)} d w_{t}
$$

subject to a constant killing rate 1 . The boundaries of $\left(\tilde{x}_{t} ; t \geq 0\right)$ are now both entrance boundaries and so $\tilde{\tau}_{x}=\infty$. $\left(\tilde{x}_{t} ; t \geq 0\right)$ is not absorbed at the boundaries. The stopping time $\bar{\tau}_{x}$ of $\left(\tilde{x}_{t} ; t \geq 0\right)$ is just its killing time $\tilde{\tau}_{x, \text { a }}$ which is mean 1 exponentially distributed, independently of the starting point $x$. Indeed,

$$
\begin{aligned}
\mathbf{P}\left(\tilde{\tau}_{x, \partial}>t\right) & =\int_{0}^{1} \bar{p}(x ; t, y) d y=\frac{1}{\alpha(x)} \int_{0}^{1} \alpha(y) p(x ; t, y) d y \\
& =\frac{1}{x(1-x)} \sum_{k \geq 1} b_{k} e^{-\lambda_{k} t} u_{k}(x) \int_{0}^{1} y(1-y) v_{k}(y) d y \\
& =\sum_{k \geq 1} b_{k} e^{-\lambda_{k} t} v_{k}(x) \int_{0}^{1} u_{k}(y) d y=6 e^{-t} \frac{1}{6}=e^{-t},
\end{aligned}
$$

recalling $x(1-x) v_{k}(x)=u_{k}(x)$ and observing $\int_{0}^{1} u_{k}(y) d y=0$ if $k \geq 2$.

As time passes, killing of $\tilde{x}_{t}$ occurs, and given killing will never occur in the future, $\tilde{x}_{t} \stackrel{d}{\rightarrow} \tilde{x}_{\infty}$ a random variable with density $6 y(1-y)$ on $[0,1]$ which is a beta $(2,2)$ density. In this selection of paths procedure, the conditional density of $\left(\widetilde{x}_{t} ; t \geq 0\right)$ given $\widetilde{\tau}_{x, \partial}=\infty$ is indeed $\tilde{p}(x ; t, y)=e^{t} \bar{p}(x ; t, y)$, where $\bar{p}(x ; t, y)=(y(1-y) /(x(1-x))) p(x ; t, y)$. Therefore,

$$
\tilde{p}(x ; t, y)=\sum_{k \geq 1} b_{k} e^{-\left(\lambda_{k}-1\right) t} \frac{u_{k}(x)}{x(1-x)} y(1-y) v_{k}(y)
$$

Recalling $u_{1}(x)=x(1-x), v_{1}(y)=1$ and $b_{1}=6$, we get $\tilde{p}(x ; t, y) \underset{t \rightarrow \infty}{\rightarrow} 6 y(1-y)$, regardless of the initial condition $x$. This is the beta $(2,2)$ limit law of the $Q$-process of the neutral WF diffusion.

(iii) Selection of WF sample paths with large sojourn time density at $y$. Assume now that $\alpha$ solves $-G(\alpha)=\delta_{y}(x)$ if $x \in I$ and so $\alpha(x)=: \mathfrak{g}(x, y)$. Using the Green function of the neutral WF model, the transition probability density of $\left(\bar{x}_{t} ; t \geq 0\right)$ is

$$
\begin{gathered}
\bar{p}(x ; t, y)=\frac{y}{x} p(x ;, y) \quad \text { if } x<y, \\
\bar{p}(x ; t, y)=\frac{1-y}{1-x} p(x ; t, y) \quad \text { if } x>y .
\end{gathered}
$$


Thus, given $x<y(x>y),\left(\bar{x}_{t} ; t \geq 0\right)$ coincides with $\left(x_{t} ; t \geq 0\right)$ conditioned to exit in 1 ( 0 , resp.) killed at rate $\delta_{y}(x)$ when it passes through $y$, necessarily at some time.

The stopping time $\tilde{\tau}_{y}(x)$ of $\left(\tilde{x}_{t} ; t \geq 0\right)$ is just its killing time when the process is at $y$ for the last time with a geometrically number of passages at $y$ with rate 1 (or success probability $1 / 2)$.

\section{The WF Model with Selection}

Now, we focus on the diffusion process (3.8). Let $\left(v_{k}(y)\right)_{k \geq 1}$ be the Gegenbauer eigenpolynomials of the KF operator corresponding to the neutral WF diffusion (3.3) and so with eigenvalues $\lambda_{k}=k(k+1) / 2, k \geq 1$. Define the oblate spheroidal wave functions on $[0,1]$ as

$$
w_{k}^{\sigma}(y)=\sum_{l \geq 1}^{\prime} f_{k}^{l} v_{l}(y)
$$

where $f_{k}^{l}$ obey the three-term recurrence defined in [24]. In the latter equality, the $l$ summation is over odd (even) values if $k$ is even (odd).

Define $v_{k}^{\sigma}(y)=e^{\sigma y} w_{k}^{\sigma}(y)$ and $u_{k}^{\sigma}(x)=(1 / m(x)) v_{k}^{\sigma}(x)$ where $m(x)=e^{2 \sigma x} /(x(1-x))$ is the speed measure density of the WF model with selection (3.8).

The system $\left(u_{k}^{\sigma}(x), v_{k}^{\sigma}(x)\right)_{k \geq 1}$ constitute a system of eigenfunctions for the WF with selection generators $-G$ and $-G^{*}$ with eigenvalues $\lambda_{k}^{\sigma}$ implicitly defined in [24], thus with $-G\left(u_{k}^{\sigma}(x)\right)=\lambda_{k}^{\sigma} u_{k}^{\sigma}(x)$ and $-G^{*}\left(v_{k}^{\sigma}(y)\right)=\lambda_{k}^{\sigma} v_{k}^{\sigma}(y)$. The eigenfunction expansion of the transition probability density of the WF model with selection is thus, [25],

$$
p(x ; t, y)=\sum_{k \geq 1} b_{k}^{\sigma} e^{-l_{k}^{\sigma} t} u_{k}^{\sigma}(x) v_{k}^{\sigma}(y)
$$

where $b_{k}^{\sigma}=\left\langle v_{k}^{\sigma}, u_{k}^{\sigma}\right\rangle^{-1}$. The WF model with selection can be viewed as a perturbation problem of the neutral WF model (see [3]). There exist perturbation developments of $\lambda_{k}^{\sigma}$ around $\lambda_{k}$ with respect to $\sigma^{2}$, [25]. They are valid and useful for small $\sigma$.

The WF diffusion process $x_{t}$ with selection (3.8) is nonconservative, with finite hitting time $\tau_{x}$ of one of the boundaries. Following the general arguments developed in Section 2, the Yaglom limit of $x_{t}$ conditioned on $\tau_{x}>t$ is the normalized version of

$$
v_{1}^{\sigma}(y)=e^{\sigma y} w_{1}^{\sigma}(y)
$$

The limit law of $x_{t}$ conditioned on never hitting the boundaries in the remote future is the normalized version of

$$
u_{1}^{\sigma}(y) v_{1}^{\sigma}(y)=\frac{1}{m(y)} v_{1}^{\sigma}(x)^{2}=y(1-y) w_{1}^{\sigma}(y)^{2} .
$$

Because the latter conditioning is more stringent than the former, the probability mass of (5.4) is more concentrated inside the interval than (5.3). 


\section{From the WF Model with Selection to the Neutral WF Model: Doob Transform and Killing}

We shall consider the following transformation of paths for the WF model with selection. Consider the Wright-Fisher diffusion with selection (3.8): $d x_{t}=\sigma x_{t}\left(1-x_{t}\right) d t+\sqrt{x_{t}\left(1-x_{t}\right)} d w_{t}$, $x_{0}=x \in(0,1)$. For this model, $G=\sigma x(1-x) \partial_{x}+(1 / 2) x(1-x) \partial_{x}^{2}$ and both boundaries are exit.

Assume that $\sigma>0$ so that the drift term is bounded above by $f_{*}=\sigma / 4$, together with $2 f / g^{2}$ being bounded below (as a constant function here equal to $2 \sigma$ ). We are then in the general framework of the problems under study in this paper. This suggests that for some admissible choice of a superharmonic exponential function $\alpha=e^{-a x}$, the $\alpha$-Doob transform of $x_{t}$ could lead to a transformed process with bounded killing rate $d=-G(\alpha) / \alpha$. We shall choose $a=\sigma$ for its interesting features.

The transition density $p(x ; t, y)$ of $x_{t}$ admits the representation (5.2) in terms of their oblate spheroidal wave eigenfunctions. Let

$$
\alpha(x)=\mathbf{E}\left(\int_{0}^{\tau_{x}} c\left(x_{s}\right) d s\right),
$$

where $c\left(x_{s}\right)=2 x_{s}\left(1-x_{s}\right) e^{-\sigma x_{s}} / 4$ is the skewed sample heterozygosity, damped by the factor $e^{-\sigma x_{s}} / 4$. Then, $\alpha$ solves $-G(\alpha)=(1 / 2) \sigma^{2} x(1-x) e^{-\sigma x}$, with solution $\alpha(x)=e^{-\sigma x}$. In this case study, one selects sample paths of $\left(x_{t} ; t \geq 0\right)$ with large $\alpha(y)$. The dynamics of $\left(\tilde{x}_{t} ; t \geq 0\right)$ is the drift-less neutral WF dynamics $d \widetilde{x}_{t}=\sqrt{\widetilde{x}_{t}\left(1-\tilde{x}_{t}\right)} d w_{t}$, subject to quadratic killing at rate $d(x)=(1 / 2) \sigma^{2} x(1-x)$ in $I$, which is bounded above there. The boundaries of $\left(\tilde{x}_{t} ; t \geq 0\right)$ are still exit and the stopping time $\bar{\tau}_{x}$ of $\left(\tilde{x}_{t} ; t \geq 0\right)$ is $\bar{\tau}_{x}=\tilde{\tau}_{x} \wedge \tilde{\tau}_{x, \partial}$, where $\tilde{\tau}_{x}$ is its absorption time at the boundaries and $\tilde{\tau}_{x, \partial}$ its killing time. The density of the transformed process is $\bar{p}(x ; t, y)=(\alpha(y) /(\alpha(x))) p(x ; t, y)$. Its series expansion is exactly known using (5.2) for $p$.

The transformed process $\left(\bar{x}_{t} ; t \geq 0\right)$ accounts for a neutral evolution of the allele $A_{1}$ frequency subject to the additional extinction opportunity of the population itself due to killing at rate proportional to its heterozygosity. Leaving aside the fact that it can be obtained after a suitable Doob transformation, this model is of importance in population genetics: it first appeared in ([8, Page 272]) as a scaling limit of a population genetics model of recombination.

From the general study of Section 2, we obtain the following.

(i) Conditioned on $\bar{\tau}_{x}>t$, the transformed process $\left(\bar{x}_{t} ; t \geq 0\right)$ admits a Yaglom limit $\bar{q}_{\infty}$. With $v_{1}^{\sigma}$ the first oblate spheroidal eigenvector of $-G^{*}$ associated to the smallest positive eigenvalue $\lambda_{1}^{\sigma}, \bar{q}_{\infty}$ is of the product form

$$
\bar{q}_{\infty}(y)=\frac{e^{-\sigma y} v_{1}^{\sigma}(y)}{\int_{0}^{1} e^{-\sigma y} v_{1}^{\sigma}(y) d y}=\frac{w_{1}^{\sigma}(\mathrm{y})}{\int_{0}^{1} w_{1}^{\sigma}(y) d y} .
$$

This limiting probability $\bar{q}_{\infty}$ is the Yaglom limit law of $\left(\bar{x}_{t} ; t \geq 0\right)$ conditioned on the event $\bar{\tau}_{x}>t$ that both the absorption and killing times exceed $t$.

(ii) Let $\bar{G}(\cdot)=e^{\sigma x} G\left(e^{-\sigma x}\right.$.) be the infinitesimal generator of $\left(\bar{x}_{t} ; t \geq 0\right)$ with two stopping times. Now, there is a tradeoff between which of $\tilde{\tau}_{x}$ and $\tilde{\tau}_{x, \partial}$ occurs first. To solve it, we need to compute $\beta$ defined in (2.55) by $\bar{G}(\beta(x))=0$, with boundary conditions $\beta(0)=\beta(1)=1$. This is a Sturm-Liouville problem whose solution in our case is

$$
\beta(x)=\frac{e^{-\sigma x}+e^{-\sigma(1-x)}}{1+e^{-\sigma}} .
$$


The function $\beta(x)=\mathbf{P}\left(\tilde{\tau}_{x}<\tilde{\tau}_{x, \partial}\right)$ is minimal when $x=1 / 2$, with value $\beta(1 / 2)=1 /$ $(\cosh (\sigma / 2))$. This looks natural because when $\tilde{x}_{0}=x=1 / 2$, the chance to hit $\{0,1\}$ before getting killed should be the lowest.

The density of the process $\left(\bar{x}_{t} ; t \geq 0\right)$ conditioned on the event $\left\{\tilde{\tau}_{x}<\tilde{\tau}_{x, a}\right\}$ is

$$
\bar{p}_{a}(x ; t, y)=\frac{\beta(y)}{\beta(x)} \bar{p}(x ; t, y)=\frac{\beta(y)}{\beta(x)} \frac{\alpha(y)}{\alpha(x)} p(x ; t, y),
$$

and so is also explicitly known from the oblate spheroidal wave expansion (5.2) of $p(x ; t, y)$. The tail distribution of $\widetilde{\tau}_{x}$ given $\left\{\tilde{\tau}_{x}<\widetilde{\tau}_{x, \partial}\right\}$ is obtained by integrating $\bar{p}_{a}$ over $y$.

Similarly, the density of the process $\left(\bar{x}_{t} ; t \geq 0\right)$ conditioned on the event $\left\{\tilde{\tau}_{x, \partial}<\tilde{\tau}_{x}\right\}$ is

$$
\bar{p}_{\partial}(x ; t, y)=\frac{1-\beta(y)}{1-\beta(x)} \bar{p}(x ; t, y)=\frac{1-\beta(y)}{1-\beta(x)} \frac{\alpha(y)}{\alpha(x)} p(x ; t, y) .
$$

The tail distribution of $\tilde{\tau}_{x, \partial}$ given $\left\{\tilde{\tau}_{x, \partial}<\tilde{\tau}_{x}\right\}$ is obtained by integrating $\bar{p}_{\partial}$ over $y$.

The associated conditioned on absorption first process $\left(\tilde{x}_{a, t} ; t \geq 0\right)$ obeys the SDE

$$
d \tilde{x}_{a}=\tilde{f}_{a}\left(\tilde{x}_{a}\right) d t+g\left(\tilde{x}_{a}\right) d w_{t}
$$

with drift

$$
\begin{aligned}
\tilde{f}_{a}(x) & =f(x)+g^{2}(x)\left[\frac{\alpha^{\prime}}{\alpha}(x)+\frac{\beta^{\prime}}{\beta}(x)\right] \\
& =\sigma x(1-x) \frac{1-e^{\sigma(1-2 x)}}{1+e^{\sigma(1-2 x)}}
\end{aligned}
$$

and local variance unchanged $g^{2}(x)=x(1-x)$. This process has no killing part and it gets eventually absorbed at $\{0,1\}$.

In the generator $\bar{G}_{\partial}(\cdot)$ of the conditioned on killing first process, there is a killing multiplicative part which is enhanced $d /(1-\beta)>d$ and a shift in the drift, showing that the associated conditioned process $\left(\tilde{x}_{\partial, t} ; t \geq 0\right)$ exhibits a faster killing rate, but the drift shift guarantees that $\left(\tilde{x}_{\partial, t} ; t \geq 0\right)$ is not absorbed at the boundaries. With $g^{2}(x)=x(1-x)$ and $\beta$ as in (6.3), the drift takes the peculiar explicit form

$$
\tilde{f}_{\partial}(x)=-\frac{g^{2} \beta^{\prime}}{1-\beta}(x)
$$

\section{From the Neutral WF Model to the WF Model with Selection: Reciprocal Doob Transform and Branching}

We now follow the general path indicated in Section 2.7 and apply it to the particular models under concern. We, therefore, illustrate and develop the idea of a reciprocal Doob transform on the specific example of interest.

The starting point is now the neutral Wright-Fisher diffusion: $d x_{t}=\sqrt{x_{t}\left(1-x_{t}\right)} d w_{t}$, $x_{0}=x \in(0,1)$. For this model, $G=(1 / 2) x(1-x) \partial_{x}^{2}$ and both boundaries are exit. Its transition 
density $p(x ; t, y)$ admits the representation

$$
p(x ; t, y)=\sum_{k \geq 1} b_{k} e^{-\lambda_{k} t} u_{k}(x) v_{k}(y)
$$

in terms of the Gegenbauer eigenpolynomials (see Section 4.1). We shall consider the following reciprocal transformation of paths for the neutral WF model: let $\alpha(x)=e^{\sigma x}$ and consider $\bar{G}(\cdot)=\alpha^{-1} G(\alpha \cdot)$. We now have $G(\alpha)=(1 / 2) \sigma^{2} x(1-x) e^{\sigma x}$ and $b(x)=G(\alpha) / \alpha>0$.

In this case study, one selects sample paths of $\left(x_{t} ; t \geq 0\right)$ with large $\alpha(y)$. The dynamics of $\left(\tilde{x}_{t} ; t \geq 0\right)$ is easily seen to be the WF with selection dynamics

$$
d \tilde{x}_{t}=\sigma \tilde{x}_{t}(1-\tilde{x}) d t+\sqrt{\tilde{x}_{t}\left(1-\tilde{x}_{t}\right)} d w_{t}
$$

subject to quadratic branching at rate $b(x)=(1 / 2) \sigma^{2} x(1-x)$ inside $I$. We indeed have

$$
\bar{G}(\cdot)=e^{-\sigma x} G\left(e^{\sigma x} \cdot\right)=b(x) \cdot+\tilde{G}(\cdot),
$$

where $\tilde{G}$ is the KBE operator of the dynamics $\left(\tilde{x}_{t} ; t \geq 0\right)$.

With $\beta(x)=\alpha(x)^{-1}=e^{-\sigma x}$, we clearly have

$$
\bar{G}(\beta(x))=0,
$$

and $\beta$ is an harmonic function for $\bar{G}$ and as a result, Doob-transforming $\bar{G}$ by $\beta$, we get

$$
\beta^{-1} \bar{G}(\beta \cdot)=G(\cdot)
$$

which is the infinitesimal generator of the original neutral WF martingale.

The birth (creating) rate $b$ in $\bar{G}$ is bounded from above on $(0,1)$. It may be put into the canonical form $b(x)=b_{*}(\mu(x)-1)$, where $b_{*}=\max _{x \in[0,1]}(b(x))=\sigma^{2} / 8>0$ and

$$
\mu(x)=1+4 x(1-x)
$$

whose range is the interval $[1,2]$ as $x \in[0,1]$.

The density of the transformed process is $\bar{p}(x ; t, y)=(\alpha(y) /(\alpha(x))) p(x ; t, y)$. It is exactly known because $p$ is known from (7.1).

The transformed process (with infinitesimal backward generator $\bar{G}$ ) accounts for a branching diffusion (BD), where a diffusing mother particle (with generator $\widetilde{G}$ and started at $x$ ) lives a random exponential time with constant rate $b_{*}$. When the mother particle dies, it gives birth to a spatially dependent random number $M(x)$ of particles (with mean $\mu(x)$ ). If $M(x) \neq 0, M(x)$ independent daughter particles are started where their mother particle died; they move along a WF diffusion with selection and reproduce, independently and so on. 
Because $\mu(x)$ is bounded above by 2 and larger than 1 (indicating a supercritical branching process), we actually get a $\mathrm{BD}$ with binary scission whose random offspring number satisfies

$$
\begin{gathered}
M(x)=0 \text { w.p. } p_{0}(x)=0, \\
M(x)=1 \text { w.p. } p_{1}(x)=2-\mu(x), \\
M(x)=2 \text { w.p. } p_{2}(x)=\mu(x)-1,
\end{gathered}
$$

with $p_{2}(x) \geq p_{1}(x)$ (the event that 2 particles are generated in a splitting event is more probable than a single one).

For such a transformed process, the tradeoff is of a different nature: there is a competition between the boundaries $\{0,1\}$ which are still absorbing for the system of particles and the number of particles $N_{t}(x)$ in the system at each time $t$, which may grow due to branching events. The density $\bar{p}$ of the transformed process has now the following interpretation:

$$
\bar{p}(x ; t, y)=\mathrm{E}\left[\sum_{n=1}^{N_{t}(x)} p^{(n)}(x ; t, y)\right],
$$

where $p^{(n)}(x ; t, y)$ is the density at $(t, y)$ of the $n$th alive particle descending from the ancestral one (Eve), started at $x$. In the latter formula, the sum vanishes if $N_{t}(x)=0$. A particle is alive at time $t$ if it came to birth before $t$ and has not been yet absorbed by the boundaries.

Let $\bar{\rho}_{t}(x)=\int_{(0,1)} \bar{p}(x ; t, y) d y$. Then, $\bar{\rho}_{t}(x)$ is the expected number of particle alive at time $t$. We have

$$
\partial_{t} \bar{\rho}_{t}(x)=\bar{G}\left(\bar{\rho}_{t}(x)\right), \quad \bar{\rho}_{0}(x)=\mathbf{1}(x \in(0,1)) .
$$

But then, $\bar{q}(x ; t, y):=\bar{p}(x ; t, y) / \bar{\rho}_{t}(x)$ obeys the forward PDE

$$
\partial_{t} \bar{q}(x ; t, y)=\left(-\frac{\partial_{t} \bar{\rho}_{t}(x)}{\bar{\rho}_{t}(x)}+b(y)\right) \bar{q}(x ; t, y)+\widetilde{G}^{*}(\bar{q}(x ; t, y))
$$

as a result of $\partial_{t} \bar{p}(x ; t, y)=\bar{G}^{*}(\bar{p}(x ; t, y))$. We have

$$
\bar{q}(x ; t, y)=\frac{\mathrm{E}\left[\sum_{n=1}^{N_{t}(\mathrm{x})} p^{(n)}(x ; t, y)\right]}{\mathrm{E}\left[N_{t}(x)\right]}
$$

showing that $\bar{q}(x ; t, y)$ is the average presence density at $(t, y)$ of the system of particles all descending from Eve started at $x$.

Clearly, $-\left(\log \bar{\rho}_{t}(x) / t\right) \underset{t \rightarrow \infty}{\rightarrow} \lambda_{1}=1$ (and, therefore, also $-\left(\partial_{t} \bar{\rho}_{t}(x) /\left(\bar{\rho}_{t}(x)\right)\right)$, because

$$
\bar{\rho}_{t}(x)=\frac{1}{\alpha(x)} \sum_{k \geq 1} b_{k} e^{-\lambda_{k} t} u_{k}(x) \int_{0}^{1} \alpha(y) v_{k}(y) d y .
$$

The expected number of particles in the system decays globally at rate $\lambda_{1}$. 
The BD transformed process, therefore, admits an integrable Yaglom limit $\bar{q}_{\infty}$, solution to $-\widetilde{G}^{*}\left(\bar{q}_{\infty}\right)=\left(\lambda_{1}+b(y)\right) \bar{q}_{\infty}$ or $-\bar{G}^{*}\left(\bar{q}_{\infty}\right)=\lambda_{1} \bar{q}_{\infty}$. With $v_{1}(y)=1$, the first eigenvector of $-G^{*}$ associated to the smallest positive eigenvalue $\lambda_{1}=1, \bar{q}_{\infty}$ is of the product form

$$
\bar{q}_{\infty}(y)=\frac{e^{\sigma y} v_{1}(y)}{\int_{0}^{1} e^{\sigma y} v_{1}(y) d y}=\frac{\sigma e^{\sigma y}}{e^{\sigma}-1} .
$$

This limiting probability $\bar{q}_{\infty}$ is the Yaglom limiting average presence density at $(t, y)$ for the BD system of particles (it is also the ground state for $\bar{G}^{*}$ ).

There is also a natural eigenvector $\bar{\phi}_{\infty}$ of the backward operator $-\bar{G}$, satisfying $-\bar{G}\left(\bar{\phi}_{\infty}\right)=\lambda_{1} \bar{\phi}_{\infty}$ (the ground state for $\left.\bar{G}\right)$. It is explicitly here that

$$
\bar{\phi}_{\infty}(x)=\frac{1}{\alpha(x)} u_{1}(x)=e^{-\sigma x} x(1-x) .
$$

In the terminology of [26], both operators $\bar{G}(\cdot)+\lambda_{1}$. and its adjoint are critical $\left(\bar{G}(\cdot)+\lambda_{1}\right.$. $\left(\bar{G}^{*}(\cdot)+\lambda_{1} \cdot\right)$ is said to be critical if there exists some function $\bar{\phi}_{\infty} \in C^{2}\left(\bar{q}_{\infty} \in C^{2}\right.$, resp. $)$, strictly positive in $(0,1)$, such that: $\bar{G}\left(\bar{\phi}_{\infty}\right)+\lambda_{1} \bar{\phi}_{\infty}=0\left(\bar{G}^{*}\left(\bar{q}_{\infty}\right)+\lambda_{1} \bar{q}_{\infty}=0\right.$, resp. $)$ and the operators do not possess a minimal positive Green function.). In this context, the constant $\lambda_{1}$ is called the generalized principal eigenvalue. The eigenfunctions $\left(\bar{\phi}_{\infty}, \bar{q}_{\infty}\right)$ are their associated ground states.

We note that we have the $L^{1}$-product property (see [26, Subsection 4.9]).

$$
\int_{0}^{1} u_{1}(x) v_{1}(x) d x=\int_{0}^{1} \bar{\phi}_{\infty}(x) \bar{q}_{\infty}(x) d x<\infty
$$

With $p_{n}(x)=\mathbf{P}(M(x)=n)$, let

$$
l(x)=\sum_{n \geq 1} p_{n}(x) n \log n=2 \log 2 p_{2}(x) .
$$

We have the $x \log x$ condition

$$
\int_{0}^{1} l(x) \bar{\phi}_{\infty}(x) \bar{q}_{\infty}(x) d x=8 \log 2 \int_{0}^{1} x(1-x) u_{1}(x) v_{1}(x) d x<\infty .
$$

We conclude (following $[9,10]$ ) that, as a result of the condition (7.17) being trivially satisfied, global extinction holds in the following sense:

(i) $\mathbf{P}\left(N_{t}(x)=0\right) \underset{t \rightarrow \infty}{\rightarrow} 1$, uniformly in $x$,

(ii) there exists a constant $\gamma>0: e^{\lambda_{1} t}\left[1-\mathbf{P}\left(N_{t}(\mathrm{x})=0\right)\right] \underset{t \rightarrow \infty}{\rightarrow} \gamma \bar{\phi}_{\infty}(x)$, uniformly in $x$,

(iii) For all bounded measurable function $\psi$ on $I$,

$$
\mathrm{E}\left[\sum_{n=1}^{N_{t}(x)} \psi\left(\tilde{x}_{t}^{(n)}\right) \mid N_{t}(x)>0\right] \underset{t \rightarrow \infty}{\longrightarrow} \gamma^{-1} \int_{(0,1)} \psi(y) \bar{q}_{\infty}(y) d y
$$


From (i), it is clear that the process gets ultimately extinct with probability 1 . In the tradeoff between branching and absorption at the boundaries, all particles get eventually absorbed and the global BD process turns out be subcritical (even though $\mu(x)=\operatorname{E} M(x)>1$ for all $x \in$ $(0,1))$ : probability mass escapes out of $I$ although the BD survives with positive probability.

In the statement (ii), the quantity $1-\mathbf{P}\left(N_{t}(x)=0\right)=\mathbf{P}\left(N_{t}(x)>0\right)$ is also $\mathbf{P}(T(x)>t)$ where $T(x)$ is the global extinction time of the particle system descending from an Eve particle started at $x$. The number $-\lambda_{1}$ is the usual Malthus decay rate parameter. From (ii), $\bar{\phi}_{\infty}(x)$ has a natural interpretation in terms of the propensity of the particle system to survive to its extinction fate: the so-called reproductive value in demography.

(iii) with $\psi=1$ reads $\mathrm{E}\left[N_{t}(x) \mid N_{t}(x)>0\right] \underset{t \rightarrow \infty}{\rightarrow} \gamma^{-1}$ giving an interpretation of the constant $\gamma$ (which may be hard to evaluate in practise).

The ground states of $\bar{G}+\lambda_{1}$ and its adjoint are, thus, $\left(\bar{\phi}_{\infty}, \bar{q}_{\infty}\right)$ and explicit here. It is useful to consider the process whose infinitesimal generator is given by the Doob transform

$$
\bar{\phi}_{\infty}^{-1}\left(\bar{G}+\lambda_{1}\right)\left(\bar{\phi}_{\infty} \cdot\right)=\bar{\phi}_{\infty}^{-1}\left(\widetilde{G}+b+\lambda_{1}\right)\left(\bar{\phi}_{\infty} \cdot\right),
$$

because product-criticality is preserved under this transformation. The ground states associated to this new operator and its dual are $\left(1, \bar{\phi}_{\infty} \bar{q}_{\infty}\right)$. Developing, we obtain a process whose infinitesimal generator is

$$
\widetilde{G}+\frac{\bar{\phi}_{\infty}^{\prime}}{\bar{\phi}_{\infty}} g^{2} \partial_{x}=G+\frac{u_{1}^{\prime}}{u_{1}} g^{2} \partial_{x},
$$

with no multiplicative part. In our case study, we get $(1 / 2) x(1-x) \partial_{x}^{2}+(1-2 x) \partial_{x}$ adding a stabilizing drift towards $1 / 2$ to the original neutral WF model. The associated diffusion process is positive recurrent and so its invariant measure $\bar{\phi}_{\infty} \bar{q}_{\infty}=u_{1} v_{1} \propto y(1-y)$ is integrable. It is the beta $(2,2)$ limit law of the $Q$-process (see $(2.80)$ and $(4.23)$ ) for the neutral WF diffusion.

Remark 7.1. At time $t$, let $\left(\tilde{x}_{t}^{(n)}\right)_{n=1}^{N_{t}(x)}$ denote the positions of the BD particle system. Let $u(x, t ; z)=\mathrm{E}\left[\prod_{n=1}^{N_{t}(x)} z^{\psi\left(\widetilde{x}_{t}^{(n)}\right)}\right]$ stand for the functional generating function $(|z| \leq 1)$ of the measure-valued branching particle system. $u(x, t ; z)$ obeys the nonlinear (quadratic) Kolmogorov-Petrovsky-Piscounoff PDE, [27]

$$
\partial_{t} u(x, t ; z)=b_{*} \theta(x, u(x, t ; z))+\widetilde{G}(u(x, t ; z)) ; \quad u(x, 0 ; z)=z^{\psi(x)},
$$

where $\theta(x, z)=\mathbf{E}\left[z^{M(x)}\right]-z=\left(p_{2}(x) z^{2}+p_{1}(x) z\right)-z$ or

$$
\theta(x, z)=4 x(1-x) z(z-1)
$$

is the shifted probability generating function of the branching law of $M(x)$. Thus, the nonlinear part reads $b_{*} \theta(x, u(x, t ; z))=b(x) u(x, t ; z)(u(x, t ; z)-1)$, which is quadratic in $u$.

In particular, if $u(x, t):=\partial_{z} u(x, t ; z)_{z=1}=\mathrm{E}\left[\sum_{n=1}^{N_{t}(x)} \psi\left(\tilde{x}_{t}^{(n)}\right)\right], u(x, t)$ obeys the linear backward PDE

$$
\partial_{t} u(x, t)=b(x) u(x, t)+\widetilde{G}(u(x, t)) ; \quad u(x, 0)=\psi(x),
$$


involving $\bar{G}(\cdot)=\tilde{G}(\cdot)+b(x)$. The latter evolution equation is the backward version of the forward PDE giving the evolution of $\bar{p}(x ; t, y)$ as $\partial_{t} \bar{p}(x ; t, y)=\bar{G}^{*}(\bar{p}(x ; t, y)), \bar{p}(x ; 0, y)=\delta_{x}(y)$.

\section{References}

[1] T. Nagylaki, "Gustave Malécot and the transition from classical to modern population genetics," Genetics, vol. 122, no. 2, pp. 253-268, 1989.

[2] J. F. Crow and M. Kimura, An Introduction to Population Genetics Theory, Harper \& Row, New York, NY, USA, 1970.

[3] T. Maruyama, Stochastic Problems in Population Genetics, vol. 17 of Lecture Notes in Biomathematics, Springer, Berlin, Germany, 1977.

[4] W. J. Ewens, Mathematical Population Genetics: I. Theoretical Introduction, vol. 27 of Interdisciplinary Applied Mathematics, Springer, New York, NY, USA, 2nd edition, 2004.

[5] R. Durrett, Probability Models for DNA Sequence Evolution, Probability and Its Applications, Springer, New York, NY, USA, 2nd edition, 2008.

[6] J. H. Gillespie, The Causes of Molecular Evolution, Oxford University Press, New York, NY, USA, 1991.

[7] A. M. Yaglom, "Certain limit theorems of the theory of branching random processes," Doklady Akademii Nauk SSSR, vol. 56, pp. 795-798, 1947.

[8] S. Karlin and H. M. Taylor, A Second Course in Stochastic Processes, Academic Press, New York, NY, USA, 1981.

[9] S. Asmussen and H. Hering, "Strong limit theorems for general supercritical branching processes with applications to branching diffusions," Zeitschrift für Wahrscheinlichkeitstheorie und Verwandte Gebiete, vol. 36, no. 3, pp. 195-212, 1976.

[10] S. Asmussen and H. Hering, "Some modified branching diffusion models," Mathematical Biosciences, vol. 35, no. 3-4, pp. 281-299, 1977.

[11] E. B. Dynkin, Markov Processes. Vols. I, II, vol. 122 of Die Grundlehren der Mathematischen Wissenschaften, Bände 121, Academic Press, New York, NY, USA; Springer, Berlin, Germany, 1965, translated with the Authorization and Assistance of the Author by J. Fabius, V. Greenberg, A. Maitra, G. Majone.

[12] S. N. Ethier and T. G. Kurtz, Markov Processes: Characterization and Convergence, Wiley Series in Probability and Mathematical Statistics: Probability and Mathematical Statistics, John Wiley \& Sons, New York, NY, USA, 1986.

[13] P. Mandl, Analytical Treatment of One-Dimensional Markov Processes, vol. 151 of Die Grundlehren der mathematischen Wissenschaften, Academia Publishing House of the Czechoslovak Academy of Sciences, Prague, Czech Republic; Springer, New York, NY, USA, 1968.

[14] K. Itô, "On stochastic differential equations," Memoirs of the American Mathematical Society, vol. 1951, no. 4 , p. 51,1951

[15] M. Kimura, "On the probability of fixation of mutant genes in a population," Genetics, vol. 47, pp. 713-729, 1962.

[16] D. Steinsaltz and S. N. Evans, "Quasistationary distributions for one-dimensional diffusions with killing," Transactions of the American Mathematical Society, vol. 359, no. 3, pp. 1285-1324, 2007.

[17] A. J. McKane and D. Waxman, "Singular solutions of the diffusion equation of population genetics," Journal of Theoretical Biology, vol. 247, no. 4, pp. 849-858, 2007.

[18] R. C. Griffiths, "The frequency spectrum of a mutation, and its age, in a general diffusion model," Theoretical Population Biology, vol. 64, no. 2, pp. 241-251, 2003.

[19] A. Lambert, "Population dynamics and random genealogies," Stochastic Models, vol. 24, supplement 1, pp. 45-163, 2008.

[20] T. Huillet, "On Wright-Fisher diffusion and its relatives," Journal of Statistical Mechanics: Theory and Experiment, vol. 2007, no. 11, Article ID P11006, 2007.

[21] R. A. Blythe and A. J. McKane, "Stochastic models of evolution in genetics, ecology and linguistics," Journal of Statistical Mechanics, vol. 7, Article ID P07018, 2007.

[22] M. Kimura, "Stochastic processes and distribution of gene frequencies under natural selection," Cold Spring Harbor Symposia on Quantitative Biology, vol. 20, pp. 33-53, 1955.

[23] M. Kimura and T. Ohta, "The age of a neutral mutant persisting in a finite population," Genetics, vol. 75, pp. 199-212, 1973.

[24] S. Mano, "Duality, ancestral and diffusion processes in models with selection," Theoretical Population Biology, vol. 75, no. 2-3, pp. 164-175, 2009.

[25] M. Kimura, "Diffusion models in population genetics," Journal of Applied Probability, vol. 1, pp. 177$232,1964$. 
[26] R. G. Pinsky, Positive Harmonic Functions and Diffusion, vol. 45 of Cambridge Studies in Advanced Mathematics, Cambridge University Press, Cambridge, UK, 1995.

[27] A. Kolmogorov, I. Petrovsky, and N. Piscounov, "Étude de l'équation de la diffusion avec croissance de la quantité de matière et son application à un probléme biologique," Moscow University Mathematics Bulletin, vol. 1, p. 125, 1937. 


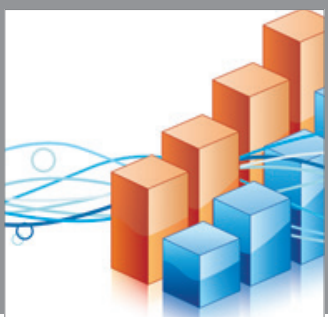

Advances in

Operations Research

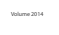

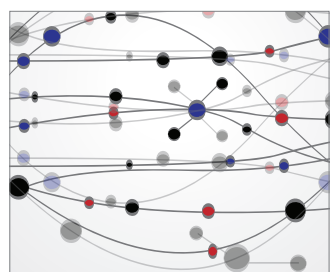

\section{The Scientific} World Journal
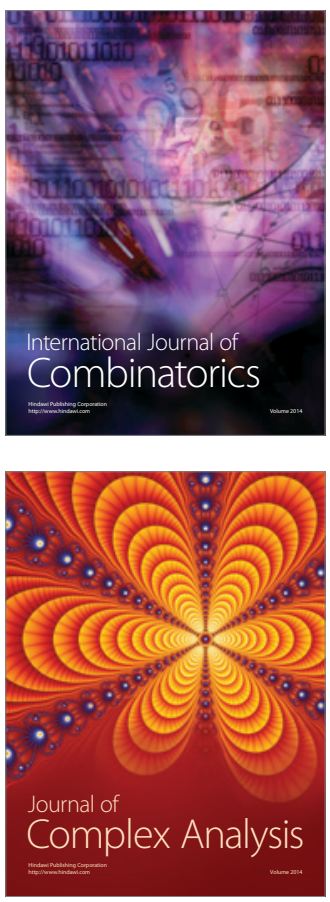

International Journal of

Mathematics and

Mathematical

Sciences
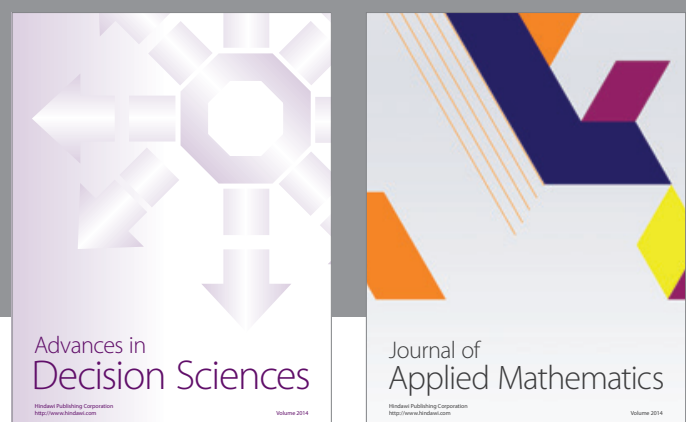

Journal of

Applied Mathematics
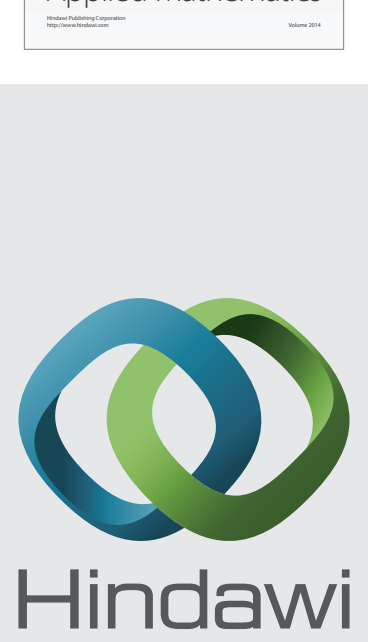

Submit your manuscripts at http://www.hindawi.com
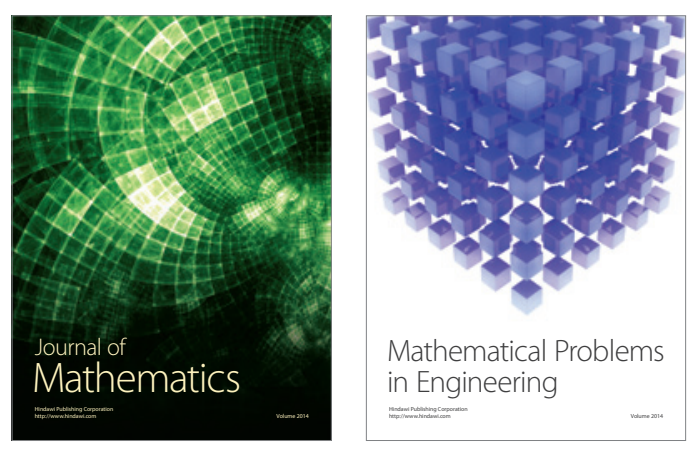

Mathematical Problems in Engineering
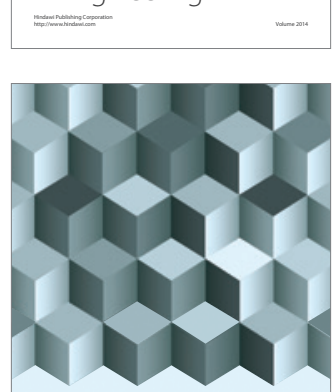

Journal of

Function Spaces
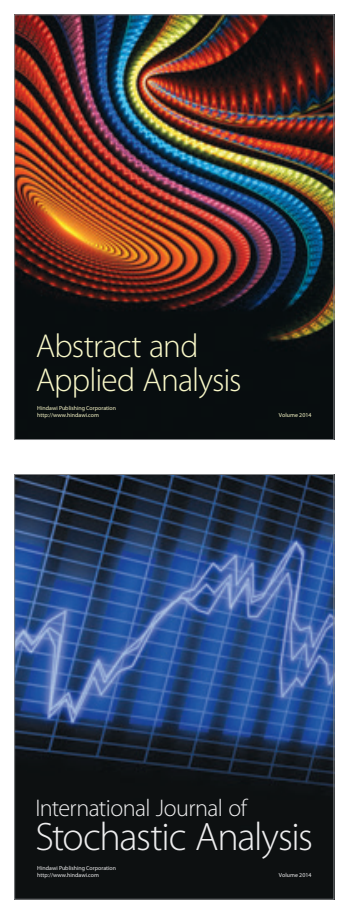

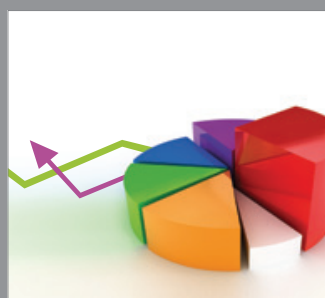

ournal of

Probability and Statistics

Promensencen
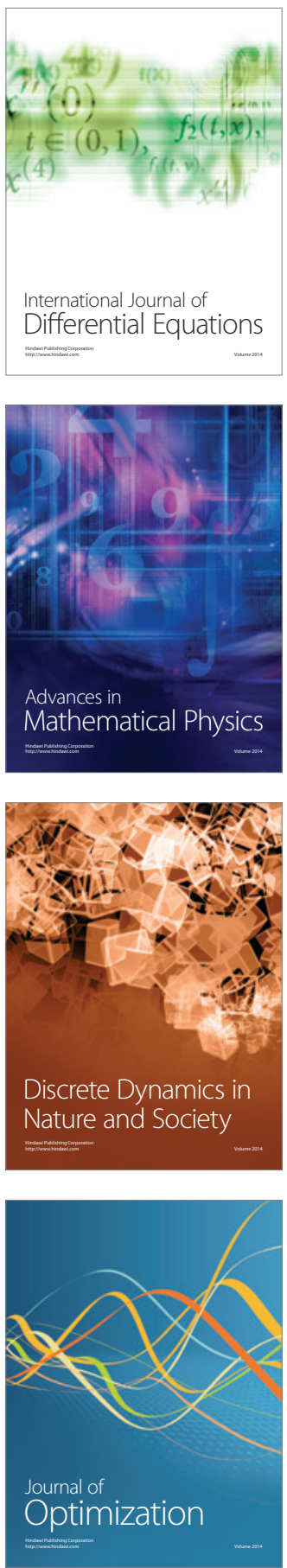